\title{
La danza come movimento ed espressione nelle immagini poetiche della Commedia di Dante
}

\author{
Claudia Cieri Via \\ Università di Roma "La Sapienza" \\ claudia.cierivia@uniroma1.it
}

\begin{abstract}
Il saggio intende interrogarsi sul ruolo della danza nella Commedia dantesca e sul rapporto fra immagine e poesia. Dall'analisi dei versi del poeta, le immagini di danza emergono attraverso le tre Cantiche ora in occorrenze o specifiche citazioni ora con allusione al loro carattere metaforico, ma spesso anche come espressione dei "moti del corpo come moti dell'animo" con riferimento alla trattatistica antica e moderna e alla luce, infine del pensiero di Aby Warburg e della elaborazione teorica del suo tempo.
\end{abstract}

Parole chiave: danza; immagine; poesia; pittura; scultura; metafora; movimento; tempo; spazio; musica; ritmo; grazia.

\begin{abstract}
The essay intends to question the role of dance in Dante's Comedy and the relationship between image and poetry. From the analysis of the poet's verses, the images of dance emerge through the three Canticles, now in occurrences or specific quotations now alluding to their metaphorical character, but often also as an expression of "motions of the body as motions of the soul" with reference to the ancient and modern treatises, and ultimately to the light of Aby Warburg's thought and the theoretical elaboration of his time.
\end{abstract}

Keywords: dance; image; poetry; painting; sculpture; metaphore; movement; time; space; music; rhythm; gracefulness. 
E come surge e va ed entra in ballo vergine lieta, sol per fare onore a la novizia, non per alcun fallo, così vid' io lo schiarato splendore venire a' due che si volgieno a nota qual conveniesi al loro ardente amore

Misesi lì nel canto e ne la rota.

(Pd. XXV, I03-Io9)

T a danza, nelle diverse culture, tempi, società presenta le sue forme di espressione e di codificazione, in alcuni casi formulate in trattati sulla danza in altri casi dando vita a rituali antichi sopravvissuti nel tempo e reinterpretati, più spesso in manifestazioni dell'immaginario collettivo dando luogo ad un processo artistico o semplicemente rappresentativo della messa in immagine della danza come movimento esteriore di movimento interiore. Nella Commedia di Dante la danza si configura per lo più come un'immagine poetica volta ad esprimere le diverse dimensioni concettuali che attraversano il suo cammino dall'Inferno al Purgatorio al Paradiso, assumendo in alcuni casi anche un valore filosofico. ${ }^{\mathrm{I}}$

Fin dall'Antichità la danza non è solo un'arte performativa ma è in rapporto con le altre arti: con la letteratura, la musica, il teatro e le arti figurative. In particolare Luciano nel suo scritto sulla danza scriveva: "La danza non è lontana dalla pittura e dalla scultura, ma sembra imitarne l'euritmia al punto che in nulla, né Fidia, né Apelle sembrano superarla" (Franzoni 2006). E' interessante questo rapporto fra le arti figurative e la danza alla luce del canone classico dell'euritmia che lega inoltre fortemente nello specifico la danza con la musica e con il movimento. In proposito vorrei riflettere sulla dinamica che coniuga il movimento della danza da una parte con il ritmo musicale che genera la danza ai fini delle codificazioni delle danze in rapporto ai contesti sociali e culturali e dall'altra con il valore espressivo dei "moti del corpo come moti dell'animo" (Cfr. Mallé 195I; Padovan 1987: 59-II2).

Il concetto di euritmia nella sua origine etimologica da rhythmos assume un significato positivo in rapporto al prefisso eũ che secondo un'antica tradizione, probabilmente pitagorica, con riferimento alla scultura, connette il ritmo alla simmetria e dunque alle proporzioni e alla commensurabilità delle parti con il tutto, alla base dell'estetica antica che informa lo statuto teorico del Canone di Policleto (Pucci 2005: 137-I52). Ma il termine euritmia va oltre il rapporto fra ritmo e proporzioni in termini razionali, matematico-geometrici. E' lo stesso Vitruvio ad evidenziare nell'euritmia una valenza estetica che implica un con-

I. "Comme toute production de l'esprit, les idées philosophiques sont elles-mêmes des fluides, c'est à dire de configurations manipulables à loisir, infiltrables partout, transformables à l'infini (Dante avait fourni à tous les artistes renaissants le modèle d'une telle liberté.)", Didi-Huberman 2015: 19. Cfr. anche Fratini 2014: 85-105. 


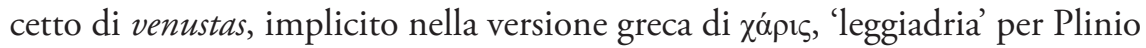
e 'grazia' per Winckelmann il quale affermava: "la grazia risiede nei gesti, si forma e si manifesta nell'atteggiamento e nel movimento del corpo" (Franzoni, 2008: 53-59; cfr. Di Rienzo 2016: 34-47). Entra dunque nell'euritmia la dimensione del movimento che attiva il processo di costruzione del disegno attraverso il tracciato della linea di contorno delle figure, della traccia che scorre sul foglio. Si tratta, secondo lo studioso Eugenio Petersen (Petersen 1917) della forma che risulta da un movimento e che si fissa a esprimere non solo la sua spazialità ma anche la sua temporalità. Il rhythmus è dunque un ossimoro che esprime il movimento nella immobilità, vale a dire una pausa del movimento, congeniale al concetto di ritmo in musica che, nel generare la danza, fissa la forma, lo schema, la figura di danza, come dell'immagine artistica.

Il leggero movimento della figura femminile scolpita da Pigmalione nel momento in cui accenna ad un passo di danza nel discendere dal piedistallo indica l'animazione della statua (Stoichita 20Io), secondo un processo inverso ma analogo a quello delle baccanti che si arrestano nel loro movimento come pietrificate dallo sguardo di medusa, fissando uno schema carico della loro espressività gestuale proprio della danza orgiastica dionisiaca nella scultura piuttosto che nella pittura o nella grafica.

Scrive Giorgio Agamben: "Il vero luogo del danzatore non è nel corpo e nel suo movimento, bensì nell'immagine come "capo di medusa", come pausa non immobile, ma carica, insieme, di memoria e di energia dinamica. Ma ciò significa che l'essenza della danza non è più il movimento ma è il tempo" (Agamben 2007: 14). Sul problema del movimento della scultura in rapporto al tempo riflette Goethe nel suo saggio sul Laocoonte che con riferimento all'arte greca individua nell'acmé il momento pregnante che esprime, nella temporalità, l'energia agente dell'immagine artistica (Goethe 1994).

Il rapporto fra la danza e l'immagine artistica era stata ben espressa anche da Plutarco che nelle Questioni Conviviali scriveva: "La danza consiste di movimenti e posizioni come il canto consiste di suoni e intervalli. Nella danza le pause sono il termine del movimento. Dunque i movimenti si chiamano passi, mentre schemata si chiamano le posizioni e le rappresentazioni verso le quali portano e nelle quali terminano i movimenti, quel momento cioè in cui i danzatori, disponendo il loro corpo nello schema di Apollo o Pan o una Baccante, rimangono in quella posizione come se fossero dipinti" (Plutarco, Questioni Conviviali, IX, I5, 747c; cit. Catoni 2008: 193).

La forte connessione fra le arti mimetiche - la danza e l'arte figurativa consiste nella possibilità per entrambe di "cristallizzare un vocabolario standard di formule iconografiche valido tanto nella lettura delle immagini scolpite o dipinte quanto di quelle danzate o rappresentate in teatro" (Catoni 2008: 193). 
La danza è dunque fra le espressioni visive la più efficace nel rapporto fra il movimento, l'impulso gestuale ed espressivo e l'immagine di danza, fra schemata e schema: "Gli schemata di danza - scrive Maria Luisa Catoni - hanno un ruolo cruciale nel fissare visualmente i modi del corpo per esprimere determinati valori" (Catoni 2008: 193). Tale passaggio dagli schemata allo schema, dallo schema, dalla forma, al rhythmos, agli schemata, coinvolge dunque il movimento, la gestualità e l'espressione, che caratterizzano diversamente i vari tipi di danza, analizzati e classificati da Aristosseno in rapporto alla tragedia, alla commedia e al dramma satiresco, da riconoscere, in mancanza di documentazione dei trattati perduti, attraverso il ritmo e il movimento del corpo (Catoni 2008: 130; Millepied-Vigarello-Martínez 2016: I25-I53).

Una ricca produzione vascolare, scultorea e pittorica testimonia il ruolo degli schemata di danze per esprimere emozioni, dolore, pathos ed ethos attraverso il ritmo delle danze satiresche da una parte e dall'altra il silenzio delle immagini velate nella coreutica antica, che caratterizzavano in particolare le tragedie di Eschilo, come commenterà Eustazio a proposito di Priapo velato:

dicono infatti che il poeta non trovando una degna rappresentazione dell'eccesso di dolore da attribuire al vecchio, lo vela e non solo lo fa silenzioso, ma non lo rende neanche visibile. Di qui, si dice, il pittore Timante di Sicione, nel dipingere l'uccisione di Ifigenia, velò Agamennone, e imitando questo stesso espediente anche Eschilo configurò Niobe e altri personaggi allo stesso modo. (Catoni 2008: 16I, n. 7I; cfr. Cieri Via 20II: IO5-I24)

Tale polarità fra pathos ed ethos troverà ulteriori elaborazioni nella successiva tradizione culturale ed iconografica della danza che ruoterà intorno alle danze in circolo dei cori angelici negli affreschi trecenteschi, alla spensieratezza delle carole narrate nel Decameron, alla spontaneità della moresca al ritmo scomposto dei canti carnascialeschi, alle varie forme dell'alta danza e alla codificazione sociale della bassa danza che si afferma nelle corti italiane fra Trecento e Quattrocento (Padovan 1987: 59-II2; Casini Ropa 2007; Millepied-ViragelloMartínez 2016: I25-153).

Alle soglie del Rinascimento saranno i modelli antichi della danza ad essere riattivati, alla luce del pensiero teorico di Leon Battista Alberti, nei trattati sulla danza di Domenico da Piacenza, di Antonio Cornazano e di Guglielmo Ebreo. Nel suo trattato De la arte di ballare e danzare (I450-60) Domenico da Piacenza indica sei elementi fondamentali dell'arte della danza: misura, memoria, agilità, maniera, misura del terreno e fantasmata ed in proposito scrive:

Dico a ti che chi del mestiere vuole imparare, bisogna danzare per fantasmata e nota che fantasmata è una prestezza corporale, la quale è mossa cum lo intellecto del mesura... facendo requie a cadauno tempo che pari aver veduto capo di medusa, come dice el poeta, cioè che facto el moto, sii tutto di pietra in quello istante e in istante metti ale come falcone che per paia mosso sia secondo la regola disopra, cioè operando mesura, memo- 
ria maniera cum mesura de terreno e aire." (Wilson, I988; cfr. Pontemoli 2009: 57-77).

Nel De arte saltandi Domenico distingue poi tra balli e basse danze, due generi compositivi completamente differenti l'uno dall'altro: i balli sono infatti sequenze movimentate e ricche di improvvisazioni, mentre le basse danze sono generalmente statiche e ben strutturate. Già in questa distinzione si possono vedere rispettivamente i germi della giga e del minuetto, destinati a dominare la scena europea sino agli inizi del XIX secolo.

Il trattato teorico sulla pittura di Leon Battista Alberti del I435 mette a fuoco il rapporto fra i moti del corpo, come espressione dei moti dell'animo, ripreso pochi anni dopo in rapporto alla danza da Guglielmo Ebreo che nel suo De practica seu arte tripudi del 1463 scriveva: "La quale virtù del danzare è una azione dimostrativa di fuori di movimenti spirituali" (Sparti 2003: 173I92; Padovan 1987: I8I-235; Padovan 1990: 25I-262).

La ripresa dei modelli antichi nell'arte del Quattrocento risponde ad una esigenza di rappresentazione del movimento nelle figure che incedono, danzano o sono spinte da impulsi emotivi oppure dal vento, come sottolinea Leonardo nel suo Trattato sulla pittura, 527:

imita quanto puoi i greci e i latini col modo di scoprire le membra quando il vento appoggia sopra di loro panni [...] ma solo farai scoprire la grossezza delle membra a una ninfa o un angello, li quali si figurino vestiti di sottili vestimenti, sospinti o impressi dal soffiare dei venti. (Camesasca 2000: 266)

Alla luce dunque di queste considerazioni ci domandiamo quale ruolo ha la danza nella Commedia dantesca e quale il rapporto fra immagine e poesia? L'immaginazione di Dante è visiva, come scriveva Thomas Eliot?

Plutarco, con riferimento al passaggio di Simonide sull'ut pictura poesis, scriveva:

il detto simonideo può essere trasposto dalla pittura alla danza. E' possibile infatti a ragione chiamare la danza una poesia silenziosa e a sua volta la poesia una danza parlante. Parrebbe infatti che nulla della poesia sia nella pittura e nulla della pittura nella poesia, né esse fanno alcun uso una dell'altra. Danza e poesia invece hanno molto in comune e partecipano una dell'altra. ...Sembrerebbe che come nella pittura, la parte poetica somigli ai colori, mentre la parte danzata alle linee che delimitano le forme. (Plutarco, Questiones Conviviales, IX, I5, 7488; cfr. Catoni 2008: 194-I95)

Da una analisi della Commedia si evincono pochi riferimenti alla danza, alcuni di ordine figurale, altri iconografici, altri di immagini poetiche, che danno 'colore' alle immagini di danza che prendono forma attraverso il movimento, come scrive Plutarco, interessanti in ogni caso, perché possono costituire, per estrapolazione, chiavi di lettura delle tre Cantiche a connotare aspetti e momenti specifici del viaggio dantesco (Venturi 2006: 15-32). 
I pochi riferimenti alla danza che si trovano nell'Inferno sono significativi essenzialmente per l'interessante articolazione del movimento espresso ora in termini di immagini poetiche ora di una dinamica realistica e drammatica a connotare la pena dei dannati in una polarità poetica espressa attraverso le immagini di danza (Nassar 1994; Brieger-Meiss-Singleton 1969).

Nel Canto VII, Dante e Virgilio si stanno avviando nel quarto cerchio degli Avari e dei Prodighi

Così scendemmo ne la quarta lacca

pigliando più de la dolente ripa

che 'l mal de l'universo tutto insacca.

(If. VII, I6-I8)

Uno spettacolo angoscioso si apre davanti ai loro occhi:

Ahi giustizia di Dio! tante chi stipa

nove travaglie e pene quant'io viddi?

e perché nostra colpa sì ne scipa?

(If. VII, I9-2I)

descritto attraverso una straordinaria metafora poetica che introduce il ruolo della danza come metafora del movimento caotico delle anime dei Dannati, che in particolare nella miniatura trecentesca di Holkam Hall si dispongono con un fluente andamento contrapposto a riproporre visivamente la metafora dei versi danteschi (Fig. I):

Come fa l'onda là sovra Cariddi, che si frange con quella in cui s'intoppa, così convien che qui la gente riddi.

Qui vidi gente più ch'altrove troppa, e d'una parte e d'altra, con grand'urli, voltando pesi per forza di poppa $\left(\right.$ If. VII, 22-27) ${ }^{2}$

Sullo stesso tema si ritorna in If. XXI, 52-57, dove nell'ottavo cerchio (Malebolge) le figure dei dannati, i barattieri, con movimenti scomposti reagiscono alle pene inflitte.

Poi l'addentar con più di cento raffi, disser: «Coverto convien che qui balli,

2. Nel passaggio citato: "così convien che qui la gente ridi" si fa riferimento ad un tipo di danza in tondo. E'interessante segnalare la coincidenza sull'osservazione del movimento delle onde legato alla danza nella biografia di Isadora Duncan: "C'est de la contemplation des vagues, quand j'étais toute petite, que m'est venue la première idée de la danse. Je tachais de suivre leur mouvement et de danser à leur rythme", I. Duncan, Ma vie, 1927, cit. in Didi-Huberman 2015: I3I e n. 27. Un'immagine visiva che può rendere bene l'immagine poetica potrebbe essere "the Great Wave" di Hokusai. Cfr. Clark 2017. 
sì che, se puoi, nascosamente accaffi.»

(If. XXI, 52-54)

Qui, in maniera drammatica, alla metafora poetica naturalistica del movimento delle onde fra Scilla e Cariddi del VII canto per dare l'immagine del movimento contrapposto dei prodighi e degli avari nel quarto cerchio, si sostituisce quella brada dei cuochi che ai loro vassalli (Fig. 2):

fanno attuffare in mezzo la caldaia

la carne con li uncin, perché non galli.

(If. XXI, 56-57)

Le immagini che illustrano i dannati, dai lussuriosi, ai violenti contro Dio agli avari e i prodighi, ai sodomiti, spesso evidenziano i movimenti scomposti di figure contorte e rovesciate che sembrano alludere ai movimenti dell'alta danza: dalla moresca alla giga, alla sarabanda, resi efficacemente nelle miniature (Fig. 3), nelle incisioni e in particolare nei disegni eseguiti da Botticelli per la prima Cantica della Commedia (Fig. 4).

Un più preciso riferimento alla danza riguarda l'episodio dedicato ai tre fiorentini, Guido Guerra, Tegghiaio Aldobrandi e Iacopo Rusticucci, i quali oggetto di una discussa interpretazione dei versi 2I-27 del canto XVI:

fenno una rota di sé tutti e trei, qual sogliono i campion far nudi e unti, avvisando lor presa e lor vantaggio, prima che sien fra lor battuti e punti; e sì rotando ciascun 'l visaggio drizzava a me, sì che 'n contraro il collo faceva ai pié continuo viaggio.

(If. XVI, 2I-27)

L'interpretazione di Sapegno a favore di una disposizione dei tre sodomiti nella figura di una danza in tondo si basa sull'immagine poetica che viene fissata nella mente del lettore dall'insistenza dell'uso de termini 'rota', 'rotare' propri della danza in tondo. (vv. 21, 25, 85). Le miniature di alcuni codici trecenteschi (Paris, BNF, ms-it. 74, I4 v. - Fig. 5) e Quattrocenteschi (Città del Vaticano, BAV, urb.lat. 365, 4I r. - Fig. 6) raffigurano i tre fiorentini che girano in tondo, sulla scorta del v. 2I "fenno una rota di sé tutti e trei», in quella sorta di danza ben colta da Iacomo della Lana che sintetizza «ellino tre feceno un ballo di loro, e sovra lo centro di tal ballo si volgeano all'usato movimento» e da Benvenuto da Imola che scrive "idest tripudium», da intendere come la sacerdotale danza romana a tre tempi (cfr. Sapegno 2004: 72). ${ }^{3}$

3. Sull'immagine di lotta si sofferma Inglese 2007: I87-188. Cfr. Terzi 2003 e Pasquino 2017. Ringrazio Gianni Pittiglio per le segnalazioni bibliografiche e per le immagini. 
Un più preciso riferimento all'alta danza si può rintracciare nel $\mathrm{X}$ canto del Purgatorio dove Dante per indicare l'atto di umiliazione di David di fronte a Dio descrive la danza dell' 'umile salmista' che "va trescando" vale a dire che esegue una danza scomposta e popolaresca, visualizzata da Botticelli in uno dei disegni della Commedia nel codice Hamilton 20I, conservato al Kupferstichkabinett di Berlino. Così la danza come la sua denudazione sono atti di umiltà, in segno di deposizione del potere ai fini di un innalzamento verso Dio (Cfr. Santarelli 2004: I09-I28; Pietrini 2000: 45-59 - Fig. 7).

Altri riferimenti alla danza si rintracciano negli ultimi Canti del Purgatorio. In particolare il passaggio dal canto XXVII al XXVIII segna la svolta del faticoso viaggio del pellegrino, riassunto dalle parole di congedo di Virgilio alla fine del XXVII. Scriveva Natalino Sapegno in proposito: "La nota malinconica e patetica è appena accennata con virile pudicizia. L'accento batte sull'importanza dello sforzo compiuto e sulla grandezza dell'acquisto che ne consegue" (Sapegno 1985: n. a v. I26).

Con l'arrivo di Dante nel Paradiso terrestre, in una sorta di ritorno all'origine, si instaura una immediata dimensione di serenità che dunque si contrappone alla dimensione angosciosa della selva oscura, peraltro agli antipodi del Paradiso terrestre, nella quale Dante si era sperso, terrorizzato dalle tre fiere in apertura della prima Cantica. Inoltre l'apparizione di Beatrice nel II Canto dell'Inferno, evocata da Virgilio come supporto per Dante nel momento di maggiore pericolo nel suo coinvolgimento nella selva oscura, trova un corrispettivo nell'arrivo di Matelda nel Paradiso terrestre. Entrambe le figure esprimono il loro ruolo attraverso l'effetto benefico della loro apparizione di figure leggiadre analogamente rappresentate nelle successive illustrazioni della Commedia come donne che volteggiano ed incedono danzando (Fig. 8).

Infatti lì Beatrice è rappresentata o descritta come la donna del dolce stilnovo, come la virtù in grado di condurre l'essere umano alla salvezza eterna. E'interessante rilevare come tale episodio e il suo significato trovi una traduzione e visualizzazione iconografica e iconologica nella miniatura lombarda dell'inizio del XV secolo del Ms. BR 39, 8 r. (Firenze, Biblioteca Nazionale - Fig. 9). Qui, accanto alla manifestazione di Beatrice che discende verso Dante dallo spazio simbolico circolare ultraterreno, dove sono contenute le tre donne, la Vergine con Lucia e Rachele, la rappresentazione delle nove Muse intorno ad Apollo, che suona un liuto, poste in cerchio tenendosi per mano stanno a simulare una danza in tondo, una carola, secondo una iconografia diffusa nel XIV secolo, configurandosi così come metafora dell'armonia, ${ }^{4}$ ad

4. Il riferimento di tale immagine è probabilmente all'invocazione di Dante all'inizio del canto: "O Muse, o alto ingegno, or m'aiutate; / $\mathrm{O}$ mente che scrivesti ciò che io vidi, / Qui si parrà la tua nobilitade", If. II, 7-9. Desidero ringraziare Lara Scanu per la ricerca dei passaggi sulla danza della Commedia. 
esprimere ulteriormente quella dimensione positiva della ispirazione poetica che permetterà a Dante di riprendere il cammino attraverso l'Inferno e il Purgatorio per arrivare al Paradiso.

Quali fioretti, dal notturno gelo chinati e chiusi, poi che l'sol l'imbianca si drizzan tutti aperti in loro stelo, tal mi fec'io di mia virtute stanca; e tanto buono ardire al cor mi corse, ch'io cominciai come persona franca;

$[\ldots]$

Tu m'hai con desiderio il cor disposto si al venir, con le parole tue, ch'i son tornato nel primo proposto. (If. II, I27-I38)

L'arrivo di Dante nel Paradiso terrestre nel Canto XXVIII del Purgatorio si connota subito come un passaggio di status, l'arrivo in un luogo privilegiato, un locus amoenus o piuttosto un giardino d'amore o il regno di Venere e della Primavera

Osserva in proposito Rosetta Migliorini Fissi che la condizione edenica è sottolineata da: "Il topos dell'alba che sancisce canonicamente una precisa corrispondenza fra il rinnovarsi del giorno - l'ora della creazione - e l'interiore renovatio del protagonista, istituendo un rapporto di piena armonia fra la creatura ed il creato secondo un ostensibile rapporto di analogia tra microcosmo (l'individuo) e macrocosmo (l'universo creato)" (Migliorini Fissi 2008: II2).

L'apparizione di Matelda assume una dimensione di magica meraviglia e si lega immediatamente ad un contesto primaverile e dunque di rigenerazione e metaforicamene e translatamente al mito di Proserpina:

e là m’apparve, si com'elli appare subitamente cosa che disvia per maraviglia tutto altro pensare, una donna soletta che si gia e cantando e scegliendo fior da fiore ond'era pinta tutta la sua via. «[...]

Tu mi fai rimembrar dove e qual era Proserpina nel tempo che perdette La madre lei, ed ella primavera.» (Pg. XXVIII, 37-5I)

Matelda dunque come Proserpina recupera il significato originario del mito antico, narrato da Ovidio nelle Metamorfosi, come quella figura che nel momento paradisiaco dell'innocenza naturalistica, mentre coglie i fiori, viene rapita da Plutone che la trascina nel mondo infernale. Tale dettaglio trova una 
certa fortuna sia nell'iconografia dei giardini d'amore che in quella dell'episodio nelle illustrazioni delle Metamorfosi di Ovidio fino al XV secolo, mentre successivamente nelle immagini prevale la scena del rapimento derivata dai modelli antichi, come nell'incisione di Albrecht Dürer.5 Matelda dunque nella posizione di Proserpina ha dato nuova vitalità alla fanciulla, alla Kore, dea della giovinezza (Fig. Io).

Il riso di Matelda infatti assume in questo contesto una connotazione dottrinale, come evidenzia Giorgio Inglese commentando il passaggio di Purgatorio XXI, con riferimento al Convivio (III, XV, 2-3) "La prima bellezza sono gli occhi, la seconda il riso" (Inglese 2016: Pg. XXXI, vv. I36-138: 379).

«Voi siete nuovi e forse perch'io rido», cominciò ella, «in questo luogo eletto. ${ }^{6}$

(Pg. XXVIII, 76-77)

Alla luce del salmo Delectasti (Salmo XCI) che 'puote disnebbiare vostro intelletto’ (Pg. XXVIII, 8I), Matelda insegna a Dante a riconoscere la bellezza del creato con ordine e misura; a questa posizione si riannodano Ugo di San Vittore, San Bonventura e San Tommaso come introibo al superiore livello conoscitivo "L'ascesa del finito intelletto umano dai visibilia terreni agli invisibilia". 7

Matelda dunque come Proserpina sta fra la dimensione infernale e quella primaverile della rinascita e in quanto tale si avvia a divenire una prefigurazione di Beatrice. In chiusura del canto infatti, Dante, dopo essersi voltato indietro verso Stazio e Virgilio, ritorna ad affiggere lo sguardo verso Matilde, ossia verso un superiore livello conoscitivo ed ontologico che condurrà a Beatrice, vale a dire ad un incomparabilmente più alto traguardo spirituale, come sarà ben visualizzato nel disegno di Botticelli (Fig. II; Migliorini Fissi 2008: 126).

Alla fine dell'Ottocento il giovane Aby Warburg, lo storico dell'arte di Amburgo, aveva riferito l'episodio dell'apparizione di Matelda al dipinto di Botticelli della cosiddetta Primavera. ${ }^{8}$ Alla Primavera e ai versi dedicati a Venere nel XXVIII Canto del Purgatorio sembra infatti essersi ispirato anche Botticelli, come hanno sottolineato recentemente alcuni studiosi che hanno

5. Bisogna aspettare la seconda metà del Cinquecento per ritrovare le due fasi del mito di Proserpina raccontato da Ovidio nel dipinto di Niccolò dell'Abate, dove in primo piano Proserpina è nella tradizionale postura del cogliere i fiori, mentre al centro campeggia l'immagine del Ratto.

6. Nell'occasione desidero ringraziare Giorgio Inglese per la segnalazione di alcuni passaggi sulla danza nella Commedia.

7. Il riferimento al Salmo Delectasti, XCI (v.8o), insegna a Dante a contemplare Dio e, oltrepassando le parvenze sensibili, a riconoscerlo nella magnificenza del creato, nella fulgente bellezza ed armonia; cfr. Ciccuto 2016: 49-80.

8. In realtà come ha sottolineato recentemente Roberto Antonelli il riferimento di Warburg ai disegni di Botticelli e ai versi della Commedia erano al XXIX del Purgatorio anziché al citato canto XXVIII, 34-69; cfr. Antonelli 2016: I3-19. 
proposto ulteriori interpretazioni del dipinto di Botticelli agli Uffizi alla luce dei versi danteschi (Barolsky 1981: I-6; Bredekamp 1996: 64 e sgg.; La Malfa 1999: 249-293; Antonelli 2016: 13-19).

Tosto che fu là dove l'erbe sono bagnate già dal'onde del bel fiume, di levar li occhi suoi mi fece dono.

Non credo che splendesse tanto lume sotto le ciglia a Venere, trafitta dal figlio fuor di tutto suo costume.

Ella ridea dall'altra riva dritta, trattando più color con le sue mani, che l'alta terra sanza seme gitta (Pg. XXVIII, 6I-69)

La descrizione dell'apparizione di Matelda sembra derivare dall'osservazione dei rilievi antichi, così presenti agli occhi di Dante, come già rivelava la descrizione ecfrastica dei rilievi scultorei dedicati all'Annunciazione e alla Giustizia di Traiano nel X canto del Purgatorio (cfr. Cieri Via 20II: 58I-6I2.; Terzoli 2016: 23-48). Sull'interesse di Dante per l'arte e le immagini artistiche ci sono diverse testimonianze che supportano il suo interesse per le descrizioni figurative attraverso le quali il poeta rende visivamente evidenti i concetti che intende enunciare tramite le similitudini, le metafore visive, le immagini poetiche che evocano atmosfere, suoni, colori, immagini naturali e mentali.

Con un riferimento alle figure all'antica dunque Dante dipinge attentamente l'incedere di Matelda attraverso una immagine di danza:

Come si volge, con le piante strette a terra e intra sé, donna che balli, e piede innanzi piede a pena mette, volsesi in su i vermigli e in su i gialli fioretti verso me, non altrimenti che vergine che li occhi onesti avvalli; e fece i prieghi miei esser contenti, sì appressando sé, che 'l dolce suono veniva a me co' suoi intendimenti. (Pg. XXVIII, 52-60)

Il riferimento all'immagine di ninfa che avanza al passo di danza trova dunque precisi esempi nei rilievi scultorei dell'antichità greco-romana, ripresi dagli artisti fiorentini del Quattrocento: dalle tre Grazie danzanti in circolo nel dipinto della cosiddetta Primavera di Botticelli (Francalanci 1992: 23-37), alla Salomé danzante di Filippino Lippi. Sull'immagine di ninfa, come topos del concetto di Pathosformel nella sua ricerca sulla sopravvivenza dell'antico, rifletteva Aby Warburg, che aveva dedicato i suoi primi studi oltre che ai dipinti 
di Botticelli per la famiglia Medici, anche alla ninfa portatrice di frutta di Ghirlandaio, esaltandone gli aspetti legati al movimento, ai gesti, al panneggio delle vesti, come ai capelli mossi dal vento anche nell' eterea immagine descritta e rappresentata da Leonardo nel disegno di Windsor; aspetti che caratterizzavano già le ricerche giovanili dello studioso di Amburgo degli anni Novanta dell'Ottocento sul movimento. Nei suoi appunti del 29 settembre del I890 infatti lo studioso scriveva:

Per conferire movimento ad una figura immota, occorre risvegliare una sequenza di immagini vissute, non una immagine unica: perdita dell'osservazione statica.... Nel caso di un abito in movimento ogni parte del contorno appariva come traccia di una persona che si muove in avanti che si segue passo passo (Müller 20II: 75; cfr. Cieri Via 2005: 63-72).

Una osservazione che anticipa di oltre dieci anni la descrizione dell'antica ninfa del rilievo pompeiano nel romanzo di Wilhelm Jensen, Gradiva: una fantasia pompeiana, pubblicato nel 1903 (Fig. I2).

Così la giovane donna non colpiva tanto per una sua bellezza plastica, piuttosto possedeva qualcosa che è raro trovare in qualche scultura marmorea: una grazia naturale, semplice virginea che sembrava infondere vita all'immagine di pietra. Vi contribuiva notevolmente il movimento in cui la giovane donna era rappresentata [...] Questo librarsi quasi in volo, congiunta alla sicurezza dell'incedere conferiva all'immagine la sua grazia specifica. Quale migliore denominazione di questa immagine ideale se non Gradiva "colei che avanza?"

La descrizione di Matelda nel XXVIII canto del Purgatorio sembra quasi un ekfrasis della Gradiva la cui riproduzione in ceroplastica era collocata nello studio di Freud, il quale, colpito dal racconto del giovane Archeologo Norbert Hanold, aveva pubblicato un saggio dal titolo: Delirio e sogni sulla Gradiva di Wilhelm Jensen, nel 1907 (Freud 1977: I13-218).

Alla immagine di ninfa Aby Warburg aveva dedicato una tavola di lavoro in occasione del progetto "Mnemosyne", l'Atlante della Memoria, dove accanto alle menadi danzanti al ritmo di una danza dionisiaca in preda ad un coinvolgimento emotivo orgiastico, una Maddalena, posta ai piedi della Croce pateticamente stravolta dalla morte di Cristo è raffigurata in un analoga postura nel rilievo bronzeo di Bertoldo di Giovanni, mentre l' immagine dell'angelo che appare al re Sigismondo quasi librandosi nella leggerezza delle sue vesti al vento è resa abilmente dal tratto sottile e vibrante di Agostino di Duccio della lastra marmorea destinata alla Cappella di S. Sigismondo nel Tempio Malatestiano di Rimini (Fig. I3).

9. Jensen, Gradiva. Fantasia pompeiana, I903, in Freud 1977, pp. I9-II2. Cfr. Cieri Via I990: pp. 25I-262. 
In proposito è interessante la definizione che darà Fritz Saxl nel 1932 della immagine di ninfa come esemplificazione di Pathosformel all'antica in rapporto alla danza:

Occorre chiarire come sia possibili che determinati tipi mantengano la loro forza pregnante attraverso i secoli. In parte tali forme devono certo la propria efficacia alla equilibrata e misurata chiarezza dei contorni con qui la grecità d'epoca classica ha riassunto tutte le precedenti forme espressive. Ma che il loro contenuto emotivo possa trasmettersi a livello generale in maniera tanto stupefacente si deve al fatto che, secondo Warburg, pur nell'approccio pieno di stile del mondo artistico dell'antichità classica risuoni ancora l'eco di quel trasporto e di quello sconvolgimento passionale che nei culti orgiastico-dionisiaci scatenavano in modi incredibili i moti espressivi del corpo... Si tratta principalmente di fenomeni di cristallizzazione, di forme espressive costanti, così come della loro trasformazione e del loro riutilizzo in diversi stadi della storia dell'umanità (Saxl 2012: I-25).

Tale immagine di danza dunque, ripresa attentamente dai modelli antichi nei dettagli dei versi citati:

Come si volge, con le piante strette

a terra e intra sé, donna che balli,

e piede innanzi piede a pena mette

è poeticamente espressa dai disegni di Botticelli per la Commedia, replicati da Aby Warburg in particolare in un disegno del 1900 che si conserva al Warburg Archive di Londra (Fig. I4). ${ }^{\text {IO }}$

Tali forme espressive dunque diventarono anche modelli della cultura letteraria e coreutica nel Quattrocento e nel Cinquecento, per trovare infine una nuova stagione alla fine dell'Ottocento con le ricerche di Maurice Emmanuel che pubblicherà nel i896 La danse grecque antique d'aprés le monument figurés. Il rinnovamento della danza in quegli anni passa attraverso i teorici del movimento come Rudolf Laban e Mary Wigman, con il contributo dei danzatori che, come Aleksander Sacharov e Clotilde van Derp, nel igıo si esibirono a Monaco in a soli di Kammertanz, ispirandosi alla scultura greca e muovendosi con grazia alla musica empatica di Tchaikosky e di Strauss, per arrivare a Loïe Fuller che interpreta la poetica del simbolo propria dell'estetica secessionista e a Isadora Duncan, che si ispira alle ninfe di Botticelli e alle linee fluenti dei corpi in movimento derivate dalle decorazioni dei vasi greci. ${ }^{\text {II }}$

Immagini di danza con caratteristiche di leggerezza e di movimento si intensificano già negli ultimi canti del Purgatorio con precisi riferimenti alle danze a tre o alle danze in circolo a sottolineare l'armonia consustanziale

Io. WIA, III. Botticelli, 39.5.2. fol. 3 r. Cfr. Didi-Huberman 20I5: I08-Io9, figg. 44-45.

II. Maining 2016 e Cieri Via 2006: 63-I36. Cfr. anche Carandini-Vaccarino I997; MillepiedVigarello-Martinez 2016. 
soprattutto alle immagini femminili, sia personaggi chiave come Matelda o Beatrice ma anche immagini di concetti astratti come nel passaggio del canto XXIX:

Tre donne in giro da la destra rota

venian danzando; l'una tanto rossa

ch'a pena fora dentro al foco nota;

l'altr' era come se le carni e l'ossa

fossero state di smeraldo fatte;

la terza parea neve testé mossa;

e or parëan da la bianca tratte,

or da la rossa; e dal canto di questa

l'altre toglien l'andare e tarde e ratte.

(Pg. XXIX, I2I-I29)

Qui le tre Virtù teologali connotate dai loro colori tradizionali il rosso, il verde e il bianco, incedono al passo di danza seguite dalle quattro Virtù cardinali (Fig. I5).

Da la sinistra quattro facean festa, in porpore vestite, dietro al modo d'una di lor ch'avea tre occhi in testa.

(Pg. XXIX, I3O-I32)

Infine nel XXXI canto del Purgatorio la danza assume la dimensione spirituale che introduce Dante al cospetto di Beatrice attraverso le Quattro Virtù cardinali (Fig. 16):

La bella donna ne le braccia aprissi; abbracciommi la testa e mi sommerse ove convenne ch'io l'acqua inghiottissi.

Indi mi tolse, e bagnato m'offerse dentro a la danza de le quattro belle; e ciascuna del braccio mi coperse.

"Noi siam qui ninfe e nel ciel siamo stelle; pria che Beatrice discendesse al mondo, fummo ordinate a lei per sue ancelle.

Merrenti a li occhi suoi, ma nel giocondo lume ch'è dentro aguzzeranno i tuoi le tre di là, che miran più profondo".

Così cantando cominciaro; e poi al petto del grifon seco menarmi, ove Beatrice stava volta a noi.

(Pg. XXXI, IOO-II4)

Le Virtù cardinali dunque a loro volta introducono il poeta alle tre Virtù teologali - "che miran più profondo" - e "danzando al loro angelico carribo" permetteranno a Dante di poter sostenere la visione di Beatrice, in un percorso 
di ascesa che secondo la struttura tomistica della Scolastica accompagnava il fedele attraverso le immagini, oltre che dei Profeti e delle Arti Liberali, delle Virtù Cardinali e Teologali rappresentate nei programmi decorativi sui portali delle Cattedrali al fine di poter accedere alla visione divina all'interno del Tempio (Seznec, 1980: I52-173).

Mentre che piena di stupore e lieta

l'anima mia gustava di quel cibo

che, saziando di sé, di sé asseta,

sé dimostrando di più alto tribo

ne li atti, l'altre tre si fero avanti,

danzando al loro angelico caribo.

"Volgi, Beatrice, volgi li occhi santi",

era la sua canzone, "al tuo fedele

che, per vederti, ha mossi passi tanti!

Per grazia fa noi grazia che disvele

a lui la bocca tua, sì che discerna

la seconda bellezza che tu cele". ${ }^{\text {I2 }}$

(Pg. XXXI, I26-I38)

Scrive in proposito Andrea Battistini:

Di là dalla figura dell'ipotiposi, che secondo i canoni della manualistica aspirava a rappresentare verbalmente qualsivoglia oggetto in modo vivido e evidente, ponendolo con immediatezza sotto gli occhi dei destinatari, le metafore e le similitudini, al pari delle parabole stilizzate che rivestivano le pareti delle chiese, fungevano da imagines agentes, simboli corporei di suggestività acuita per innescare nel modo più conveniente il meccanismo mnemotecnico. Non è un caso che la Rhetorica ad Herennium, il solo trattato di arte sermocinale che insieme con il De inventione circolasse ai tempi di Dante, dedichi una porzione considerevole dei suoi enunciati proprio all'Ars memoriae [...] Accanto alla memoria la retorica iconica interviene a favore dell'actio, ossia nella pregnanza dei gesti, per i quali può aver avuto una qualche incidenza il fascino delle figurazioni artistiche (Battistini 2008: 8-9).

Maggiore è l'intensificazione dell'uso metaforico della danza in ben 7 luoghi della terza Cantica, a segnare l'esaltazione dell'ascesa e dunque del movimento verso l'alto attraverso:

L'amor che move il sole e le altre stelle.

(Pd. XXXIII, I45)

I riferimenti alla danza nel Paradiso evidenziano il rapporto con la musica e con il canto nella cadenza ritmica e temporale, già indicata come carattere del movimento della danza nel tracciare immagini circolari proprie delle carole

I2. Come evidenzia Giorgio Inglese, con riferimento al Convivio (III, XV, 2-3): "La prima bellezza sono gli occhi, la seconda il suo riso" (Inglese 20I6: 379). 
e dei cori angelici, che avevano una certa tradizione figurativa al tempo di Dante, ed un significato legato al concetto armonico di perfezione e di unità nel senso di equidistanza di tutti i punti della circonferenza dal centro secondo il pensiero plotiniano (Fig. I7).

La prima terzina del CantoVII con l'Osanna, come espressione di lode a Dio, introduce la musicalità del canto e la leggerezza della danza delle anime beate (Fig. 18).

Così, volgendosi a la nota sua, fu viso a me cantare essa sustanza, sopra la qual doppio lume s'addua;

ed essa e l'altre mossero a sua danza, e quasi velocissime faville mi si velar di sùbita distanza.

(Pd. VII, 4-9)

Nel X Canto del Paradiso la danza che le anime dei Beati inscenano intorno a Dante e a Beatrice, i quali fungono da poli di riferimento, pur nel dare un'immagine della leggerezza aerea, è diversificata nella velocità del movimento e dalla distanza dai suddetti poli; come danzatrici che danzano in tondo al ritmo musicale, le anime dei Beati si arrestano in silenzio, bloccate nella loro formazione, "non da ballo sciolte", in uno stato di tensione fra movimento e pausa, in attesa che la musica riprenda (Fig. 19). ${ }^{13}$

Poi, sì cantando, quelli ardenti soli si fuor girati intorno a noi tre volte, come stelle vicine a' fermi poli,

donne mi parver, non da ballo sciolte, ma che s'arrestin tacite, ascoltando fin che le nove note hanno ricolte. (Pd. X, 77-8I)

L'esordio del XIII Canto è all'insegna linguisticamente e poeticamente del gioco intellettuale fra l'uso dei termini 'immagini' come immagini delle stelle "astrali geometrie luminose" che appaiono alla vista di Dante ed immagini fissate pietrificate "come ferma rupe", a segnare il passaggio dalla percezione visiva delle stelle alla necessità del fermo-immagini per poterle descrivere.

Imagini, chi bene intender cupe quel ch'i' or vidi — e ritegna l'image, mentre ch'io dico, come ferma rupe quindici stelle che ' $n$ diverse plage lo ciel avvivan di tanto sereno che soperchia de l'aere ogne compage; imagini quel Carro a cu' il seno

I3. Per corrispettivi riferimenti alla danza cfr. Inglese 20I6a: I44, vv. 79-8I. 
basta del nostro cielo e notte e giorno, sì ch'al volger del tempo non vien meno; ${ }^{14}$ (Pd. XIII, I-9)

Dante ricorre ancora alla metafora poetica della danza nel passaggio sulle formazioni circolari dei beati come stelle lucenti, assimilabili, attraverso il processo di mitologizzazione delle immagini astrali, alla doppia corona di Arianna (Fig. 20; Panofsky - Saxl 2009):

imagini la bocca di quel corno che si comincia in punta de lo stelo a cui la prima rota va dintorno, aver fatto di sé due segni in cielo, qual fece la figliuola di Minoi allora che sentì di morte il gelo; e l'un ne l'altro aver li raggi suoi, e amendue girarsi per maniera che l'uno andasse al primo e l'altro al poi; e avrà quasi l'ombra de la vera costellazione e de la doppia danza che circulava il punto dov' io era (Pd. XIII, IO-20)

Nei versi subito successivi, attraverso la metafora del canto e della musica con riferimento a Bacco e ad Apollo e ai canti in loro onore, che riflettono la contrapposizione fra il concitato ritmo dionisiaco e l'armonica musica apollinea, si introduce una comparazione oppositiva con i Misteri di Fede, in una forma di polarità analoga a quella sulla quale rifletterà Aby Warburg a proposito dell' apollineo e del dionisiaco alla luce del pensiero di Nietzsche nella Nascita della Tragedia (Busch 2009: 203-216).

Lì si cantò non Bacco, non Peana, ma tre persone in divina natura, $\mathrm{e}$ in una persona essa e l'umana. (Pd. XIII, 26-29)

Qui sinteticamente in soli due versi Dante esprime i primi due misteri di fede: l'Unità della Trinità di Padre, Figlio e Spirito Santo, e la doppia natura, umana e divina, di Cristo nell'Incarnazione del Verbo.

Nella conclusione di questo lungo ed intenso passaggio il canto e la danza circolare portano dunque a compimento la misura, il tempo ritmico del loro musicale accordo, esprimendo con la poesia in modo teologico il mistero inesprimibile in termini razionali dell'Unità e della Trinità. Così canto e danza si

I4. Cfr. In proposito sull'uso dei termini "immagini" e "image" i commenti di Inglese 20r6: 306 , nota vv. 25-26. 
arrestano simultaneamente per dar luogo alla parola, al Verbo, con il discorso di San Tommaso.

Compié 'l cantare e 'l volger sua misura; e attesersi a noi quei santi lumi, felicitando sé di cura in cura.

Ruppe il silenzio ne' concordi numi

Poscia la luce in che mirabil vita

Del poverel di Dio narrate fumi.

(Pd. XIII, 28-33)

Nel Canto successivo come la danza in cerchio dei Beati, al suono della musica

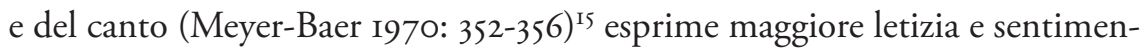
ti, in quella corrispondenza fra i moti del corpo e i moti dell'animo che sarà codificata dalla trattatistica quattrocentesca, ${ }^{16}$ così l'Orazione di Tommaso conferisce nuova e ulteriore gioia (Fig. 2I).

Come, da più letizia pinti e tratti, a la fiata quei che vanno a rota levan la voce e rallegrano li atti, così, a l'orazion pronta e divota, li santi cerchi mostrar nova gioia nel torneare e ne la mira nota.

(Pd. XIV, I9-24)

Procedendo nella ascesa, la poesia di Dante diviene sempre più simbolica ed astratta; attraverso una similitudine col volo degli uccelli che esprimono la loro gioia per un abbondante pasto, tracciando nel cielo forme circolari o geometriche; così le anime beate volano nel cielo danzando al ritmo del loro canto per arrestarsi, tacendo, al momento di formare lettere e segni. E' interessante ricordare la analogia indicata nel rapporto della danza con la pittura fra il movimento della danza e l'arresto, la pausa, a configurare una figura di danza, come un'immagine artistica, ma anche un segno, e in questo caso, lettere, D I L, ad esprimere un alto concetto: Diligite Iustitiam, che apre il primo libro dei Proverbi. L'immagine dunque visualizza la parola e la parola l'immagine, quasi in una forma di "visibile parlare" (Fig. 2I; Ciocciola 1989: 9-77; Terzoli 2016: 44; Cieri Via, in corso di stampa).

E come augelli surti di rivera, quasi congratulando a lor pasture, fanno di sé or tonda or altra schiera,

sì dentro ai lumi sante creature

15. Sul ruolo della musica cfr. Meyer-Baer 1970: 352-356.

I6. "La quale virtù del danzare è una azione dimostrativa di fuori di movimenti spirituali" scriverà Guglielmo Ebreo nel suo Trattato, De practica seu arte tripudi, del I463 (Paris, Bibliothèque Nationale, f. it. 973) 
volitando cantavano, e faciensi

or $\mathrm{D}$, or $\mathrm{I}$, or $\mathrm{L}$ in sue figure.

Prima, cantando, a sua nota moviensi;

poi, diventando l'un di questi segni,

un poco s'arrestavano e taciensi

(Pd. XVIII, 73-8I)

Scrive in proposito Andrea Battistini «Sopratutto nel Purgatorio e nel Paradiso celeste i segni tangibli e concreti risultano sempre simboli enigmaticamente speculari di una realtà ultraterrena, da ricostruire con l'allegoresi teologica" (Battistini 2008: 5-II).

Alla danza dunque Dante affida quelle immagini poetiche di elevazione spirituale delle anime dei Beati disposte in cerchi rotanti, che come sfere intorno al proprio asse, fiammeggiando in guise di comete, sono assimilabili alle ruote dentate degli orologi che scandiscono la velocità del tempo in rapporto alla loro diversa posizione e dunque grandezza (Fig. 22).

Così Beatrice; e quelle anime liete

si fero spere sopra fissi poli,

fiammando, volte, a guisa di comete.

E come cerchi in tempra d'orïuoli

si giran sì, che 'l primo a chi pon mente

quïeto pare, e l'ultimo che voli;

così quelle carole, differente-

mente danzando, de la sua ricchezza

mi facieno stimar, veloci e lente.

(Pd. XXIV, $\mathrm{II}-\mathrm{I} 8)$

Tempo e spazio sono dunque le due dimensioni che caratterizzano la danza attraverso le quali poter misurare la velocità dei movimenti della carola delle anime dei Beati ai fini di esprimere la maggiore o minore intensità di gioia $\mathrm{e}$ di beatitudine. ${ }^{17}$

Di quella ch'io notai di più carezza

vid' io uscire un foco sì felice,

che nullo vi lasciò di più chiarezza;

e tre fiate intorno di Beatrice

si volse con un canto tanto divo,

che la mia fantasia nol mi ridice."

$(P d$. XXIV, I8-24)

17. Interessante è il riferimento al concetto filosofico di movimento in Aristotile a cui Dante fa riferimento nel Convivio IV, II, 6: "Lo tempo secondo che dice Aristotile nel quarto della Fisica è 'numero di movimento secondo prima e poi [...]". Sulla valenza positiva della danza nell'Antico Testamento è esemplificativo il passaggio daI Salmo, I50,3-4, dove la scena finale inneggia a Dio attraverso la musica e la danza: "Lodatelo al suono delle trombe, lodatelo sull'arpa e sulla cetra; lodatelo coi timpani e con la danza". Cfr. Pietrini 2000: 45. 
Una gioia e una beatitudine - come scrive Dante - che è inesprimibile attraverso la poesia. E' molto suggestiva la similitudine fra il parlare, in contrapposizione al silenzio, e il colore troppo vivo delle immagini pittoriche, per le quali il pittore fa uso delle pieghe nei panneggi delle vesti e attraverso l'ombra e il chiaroscuro rende la pittura silenziosa.

Però salta la penna e non lo scrivo:

ché l'imagine nostra a cotai pieghe,

non che 'l parlare, è troppo color vivo.

(Pd. XXIV, 26-27)

Le immagini di danza come le immagini artistiche si pongono nella Commedia "al servizio di un procedimento fondato sulla manifestatio, con cui si rendevano palpabili ed espliciti l'ordine e la logica di un pensiero, soprattutto di natura teológica" (Battistini 2008: 8).

Ancora alla danza, come espressione di leggerezza e di grazia, è ispirata la metafora poetica degli ultimi versi del XXV del Paradiso:

E come surge e va ed entra in ballo vergine lieta, sol per fare onore ala novizia, non per alcun fallo,

così vid' io lo schiarato splendore venire a' due che si volgeno a nota, qual conveniesi al loro ardente amore.

Misesi lì nel canto e ne la rota; e la mia donna in lor tenea l'aspetto, pur come sposa tacita e immota. (Pd. XXV, IO3-III)

con la quale il poeta introduce il tema dell'amore attraverso la figura di San Giovanni: l'amore come il motore che spinge Dante ad una elevazione verso la visione accecante della Divinità (Fig. 23).

Qual'è colui ch'adocchi e s'argomenta di veder eclissare lo sole un poco, che, per veder, non vedente diventa; tal mi fec'io a quell'ultimo foco mentre che detto fu: "Perché t’abbagli per veder cosa che qui non ha loco?" (Pd. XXV, II8-I23)

Commenta in proposito Giorgio Inglese: "Nel discorso mistico l'accecamento simbolizza di regola un progresso nell'ascesa verso la verità", citando Dionigi l'Aeropagita: "Preghiamo di trovare, in questa tenebra luminosissima, e, mediante la privazione della vista e della conoscenza, poter vedere e conoscere ciò che sta oltre la visione e la conoscenza" (Inglese 20I6a: 318, n. II8-I20), per concludere sui limiti della condizione umana nei versi che chiudono il XXV 
canto, non prima di una ulteriore immagine poetica legata alla danza circolare dei tre Apostoli:

A questa voce l'infiammato giro si quietò con esso il dolce mischio che si facea nel suon del trino spiro [...]

Ahi quanto nella mente mi commossi, quando mi volsi per veder Beatrice, per non poter veder, benchè io fossi, presso di lei, e nel mondo felice. (Pd. XXV, 130-I39)

Con la chiusura del XXV canto del Paradiso si pongono le premesse per l'espansione della filosofia della luce che, attingendo a Dionigi l'Aeropagita, a Sant'Agostino, a San Bonaventura, concluderà la terza cantica e l'itinerarium mentis in deum del sommo poeta.

Qual' è il geometra che tutto s'affige per misurar lo cerchio e no ritruova, pensando quel principio ond'egli indige,

tal ero io a quella vista nova: veder voleva come si convene l'imago al cerchio e come vi s'indova; ma non era da ciò le proprie penne, se non che la mia mente fu percossa da un fulgore in che sua voglia venne.

All'alta fantasia qui mancò possa, ma già volgea il mio disio e l'velle si come rota che igualmente è mossa,

l'amor che move il sole e le altre stelle. (Pd. XXXIII, I33-I45) 


\section{IMMAGINI}
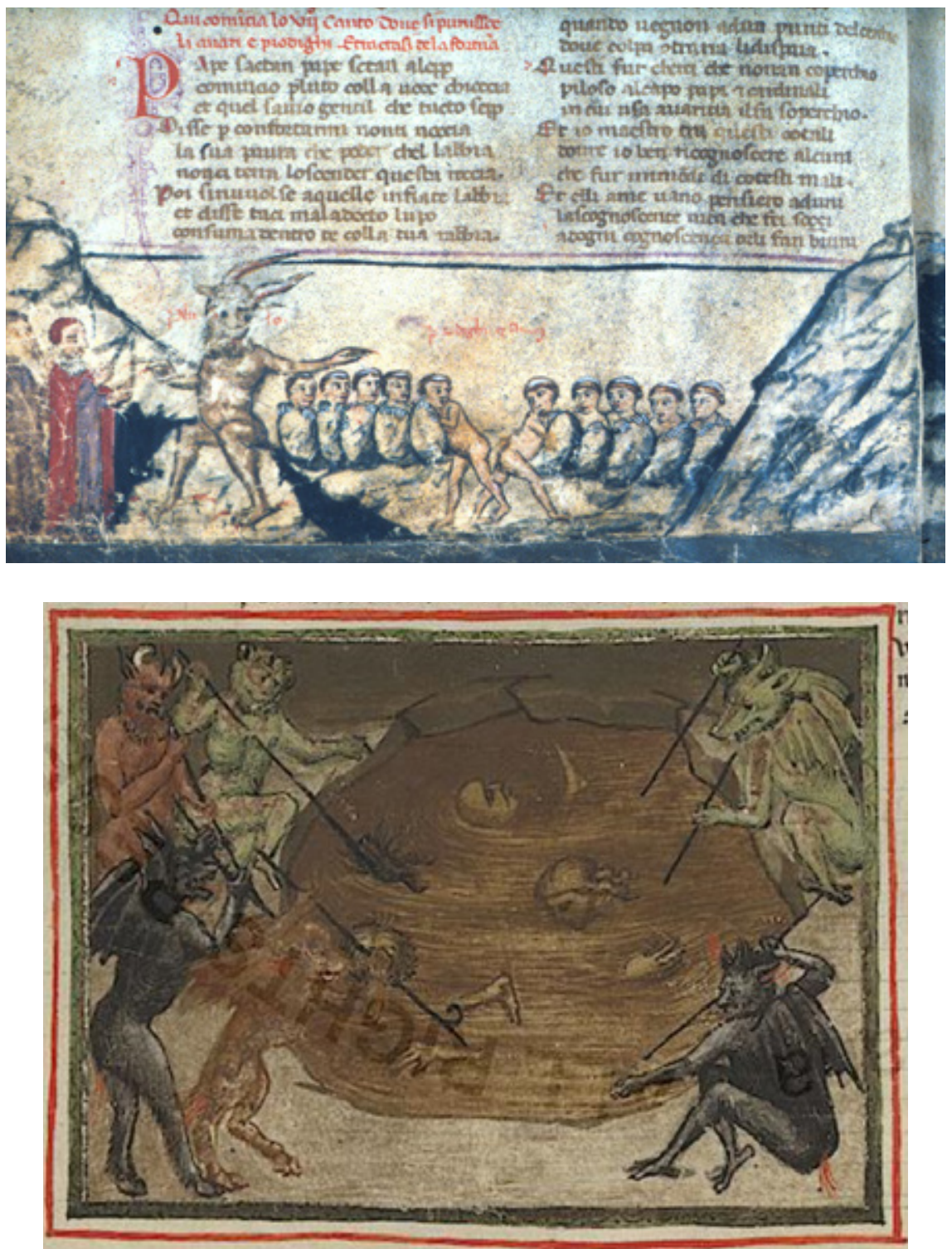

Fig. I. If. VII 24 , Holkham Hall Ms. 514, I370 ca. Oxford, Bodleian Library.

Fig. 2. If. XXI 52-54, Vat. Lat. 4776, 72r, Città del Vaticano, Biblioteca Apostolica Vaticana. 

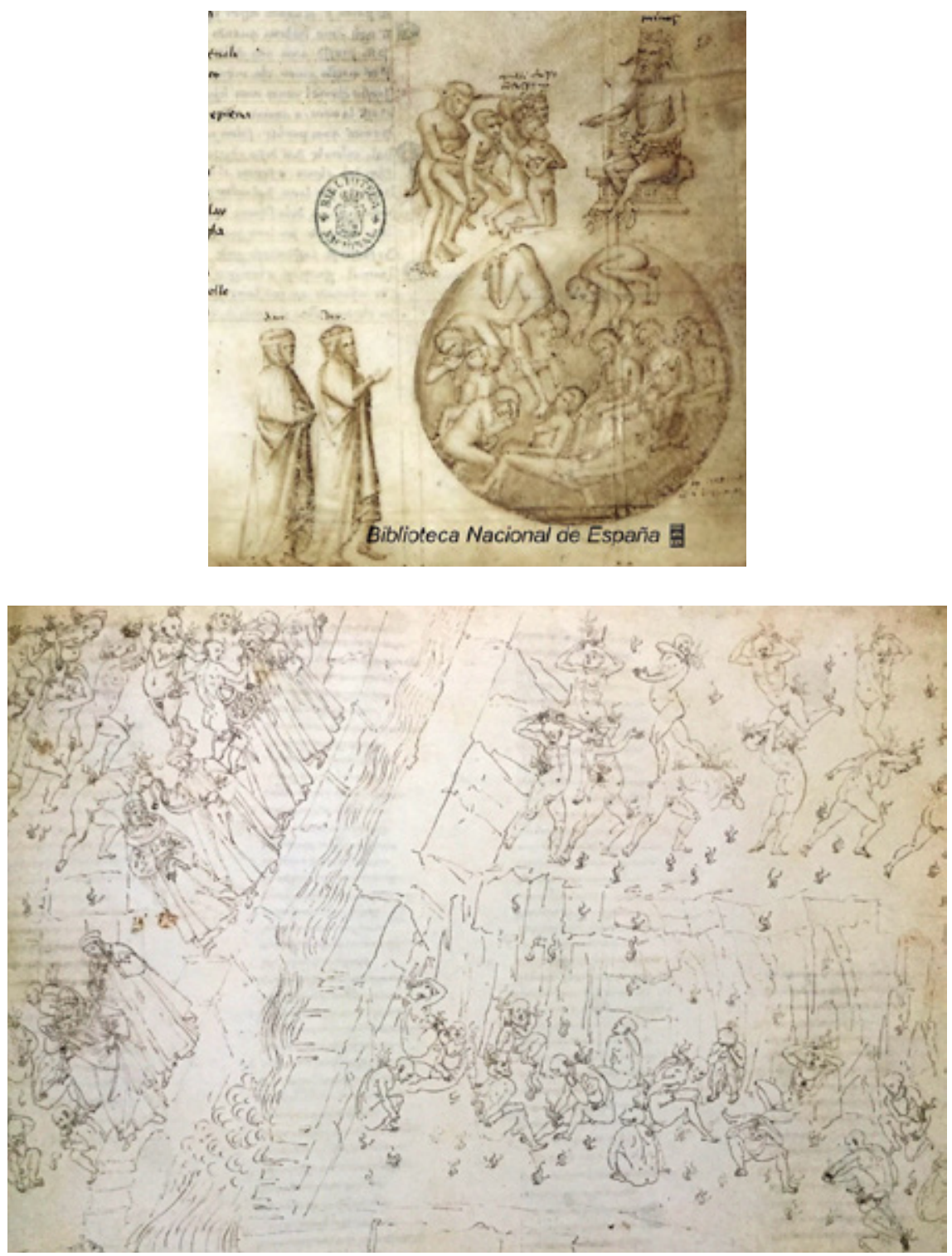

Fig. 3. If. V Io, Minosse e i lussuriosi, Madrid, Biblioteca Nacional, Ms. I0057, in r.

Fig. 4. If. XVI, I violenti contro l'ordine divino, Botticelli, Ms. Reginensis lat. I896, c.98 v. Città del Vaticano, Biblioteca Apostolica Vaticana, ms. Reginensis lat. I896, c. 98 v. 

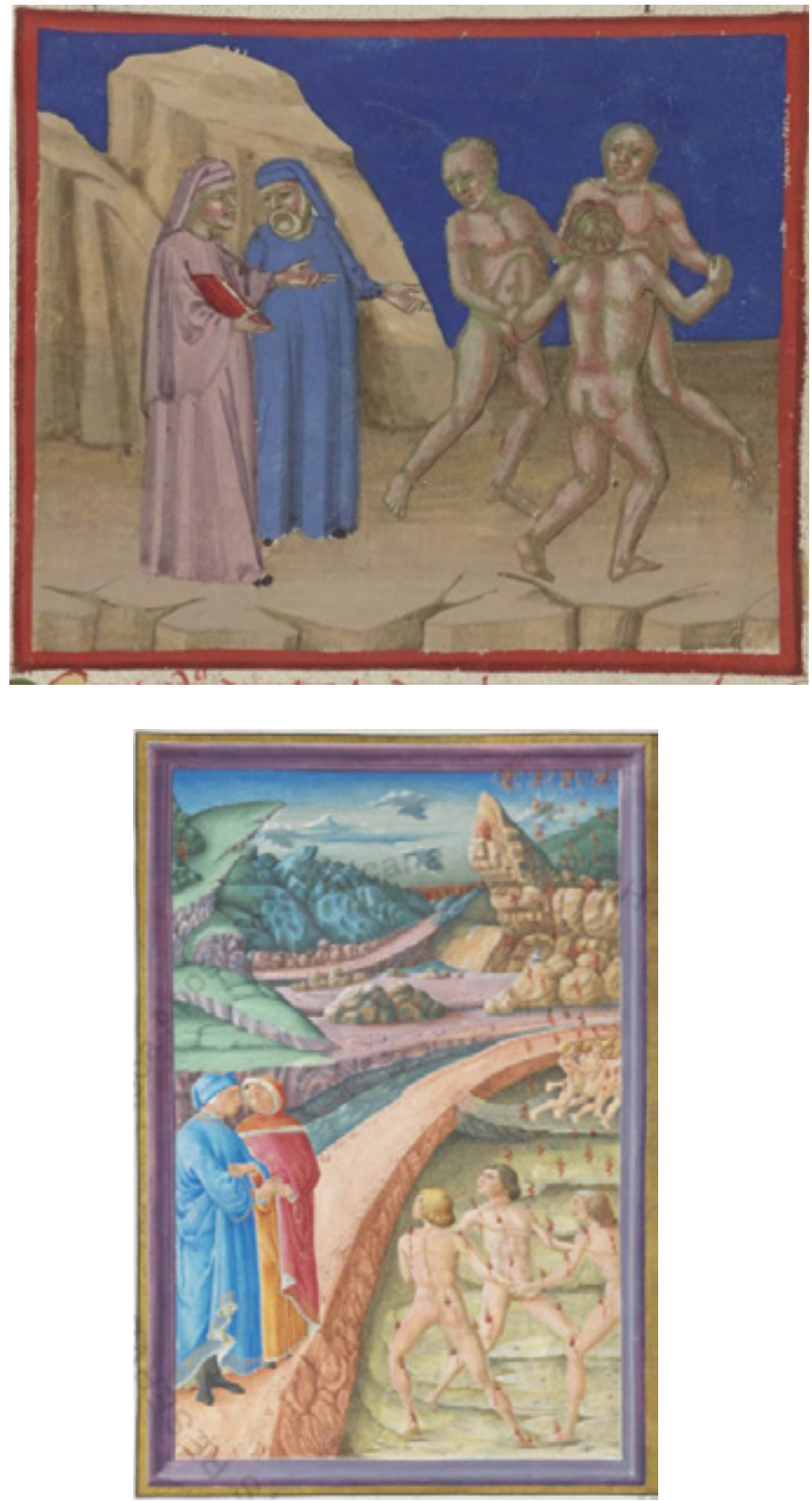

Fig. 5. If. XVI 2I-27, Paris, BNF, ms-it. 74, I4 v

Fig. 6. If. XVI 21.27, Città del Vaticano, Biblioteca Apostolica Vaticana, Ms. Urb.lat. 365, 4I r. 

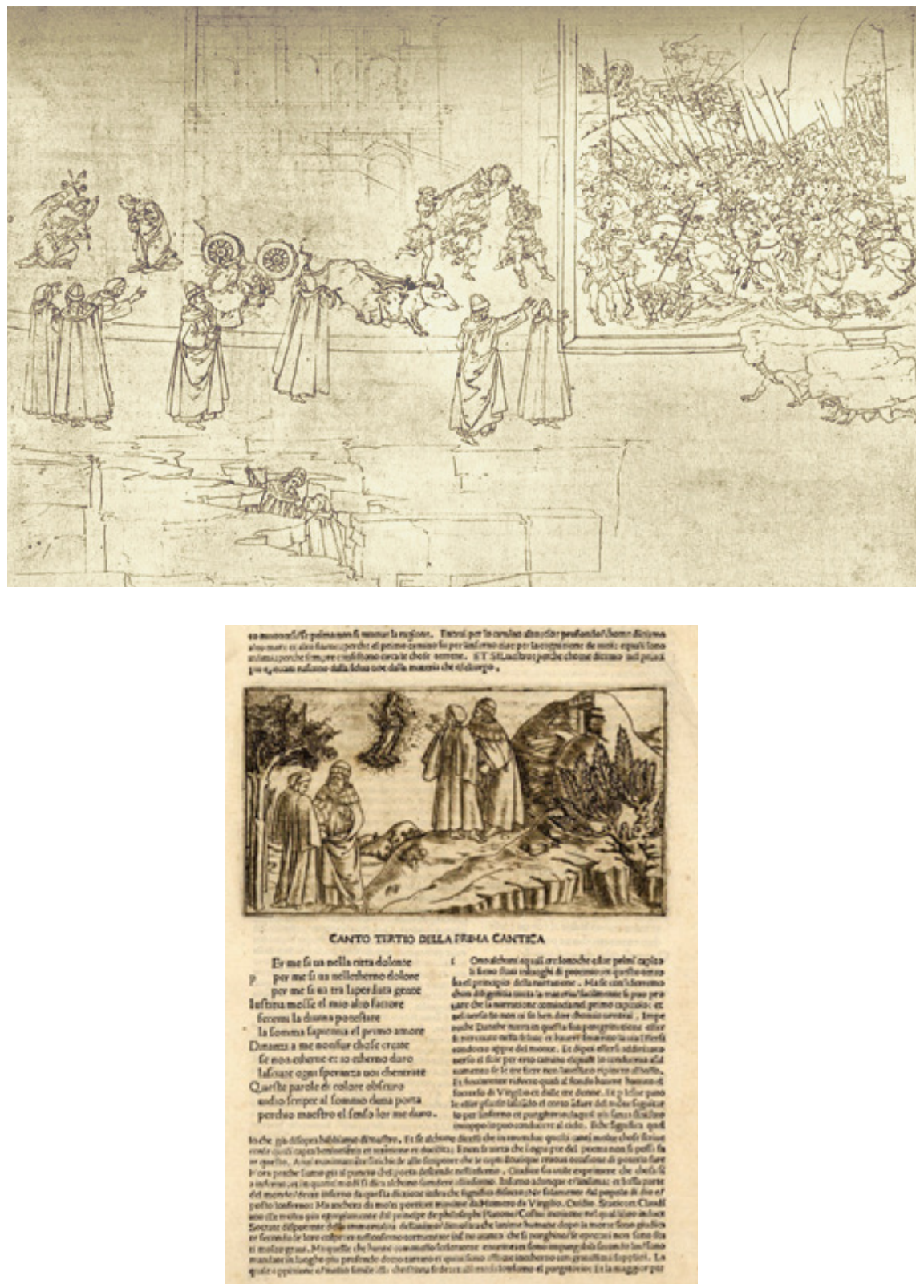

Fig. 7. Pg. X. 64-66, Botticelli, La danza di David, Ms. Hamilton 20I, Berlino Kupferstichkabinett. Fig. 8. If. II, 52 e ss., Baccio Baldini, Commento di Cristoforo Landino, ed. Firenze I48I, Londra, British Museum, incisione. 

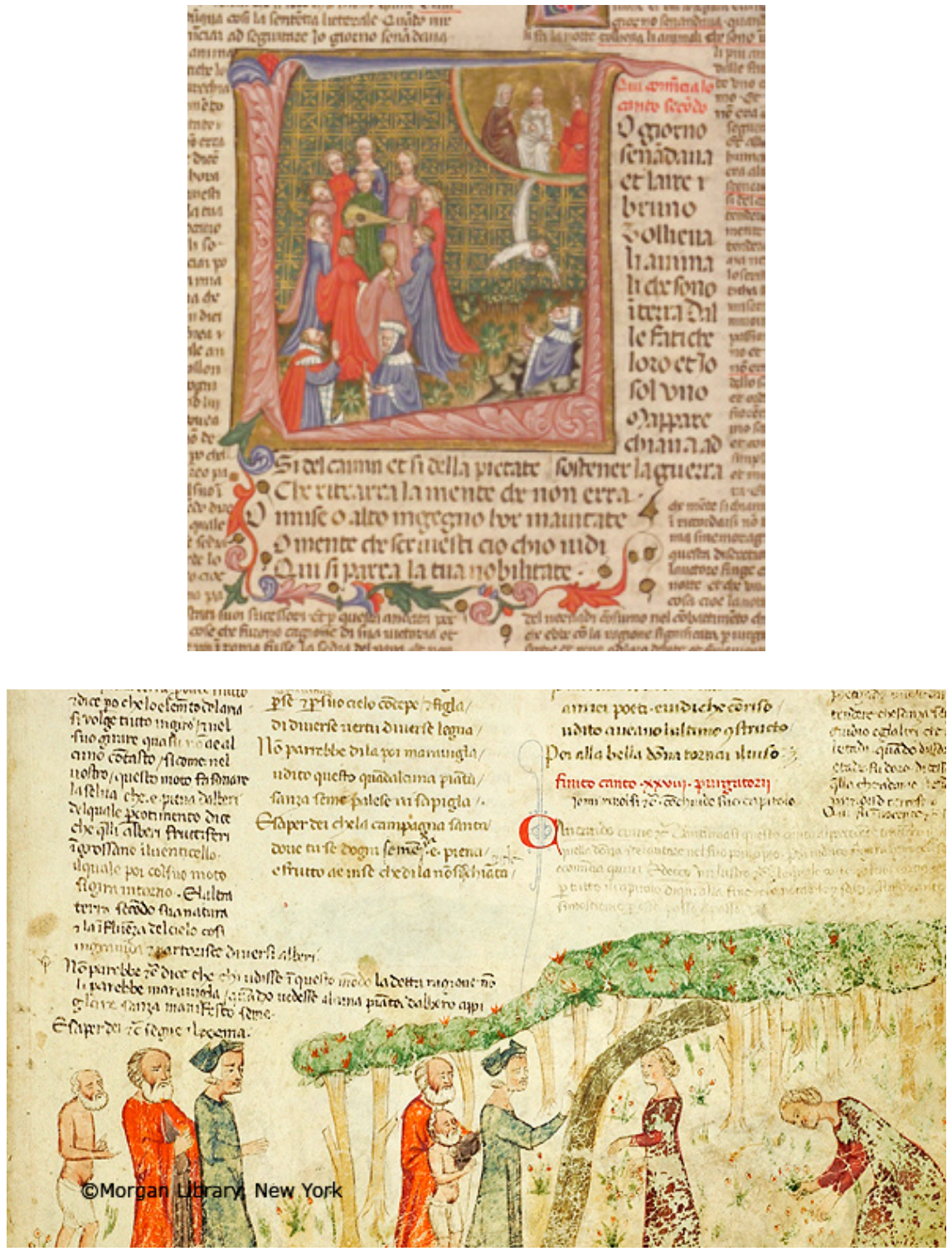

Fig. 9. If. II, I-9, Ms. BR 39, 8r., Firenze, Biblioteca Nazionale.

Fig. Io. Pg. XXVIII,37-5I, Ms. 676, f. 83, New York, Morgan Library. 

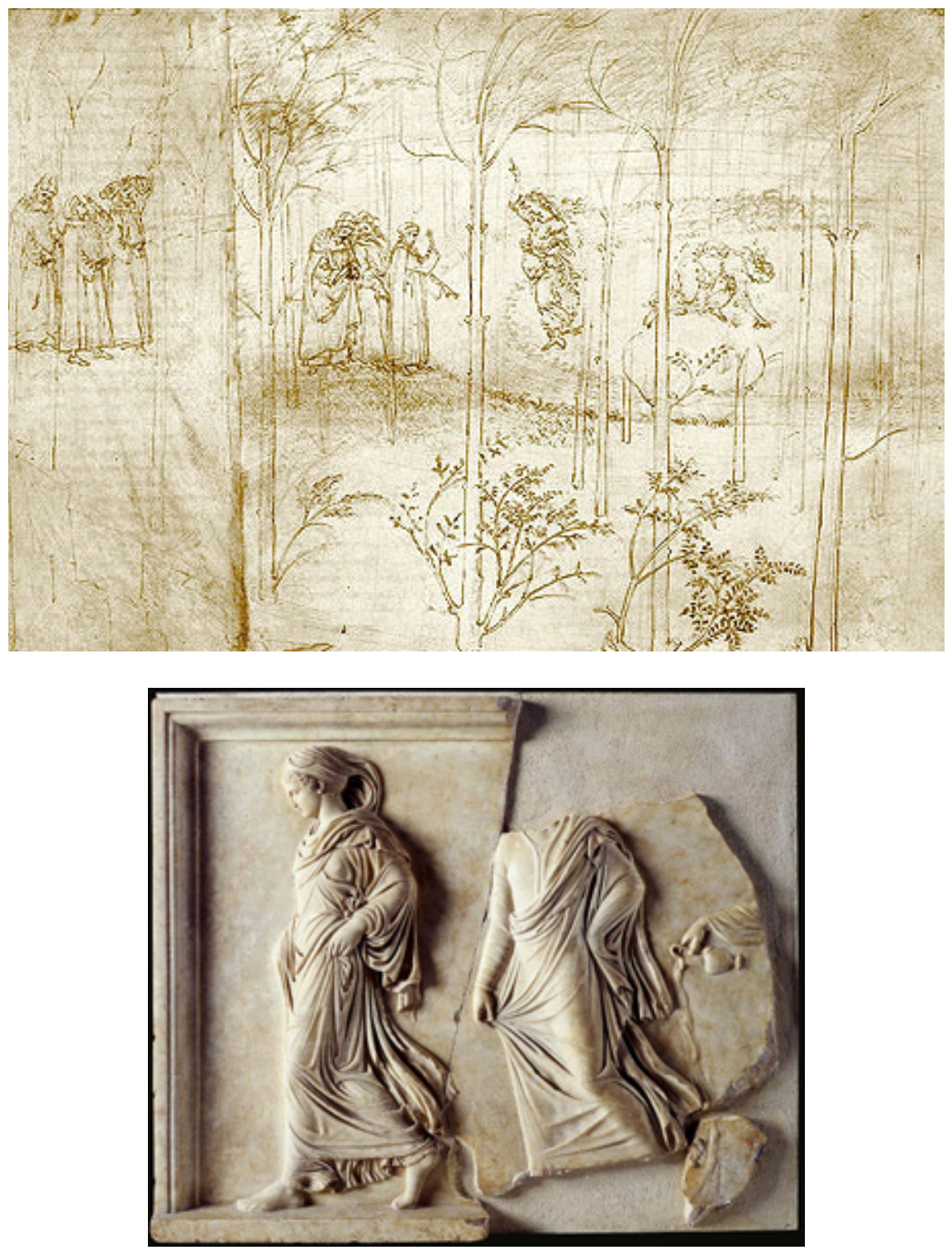

Fig. II. Pg. XXVIII, I45-I48, Botticelli, ms. Hamilton 207, Berlino Kupferstichkabinett. Fig. I2. Gradiva, Museo Chiaramonti, Musei Vaticani, Città del Vaticano. 

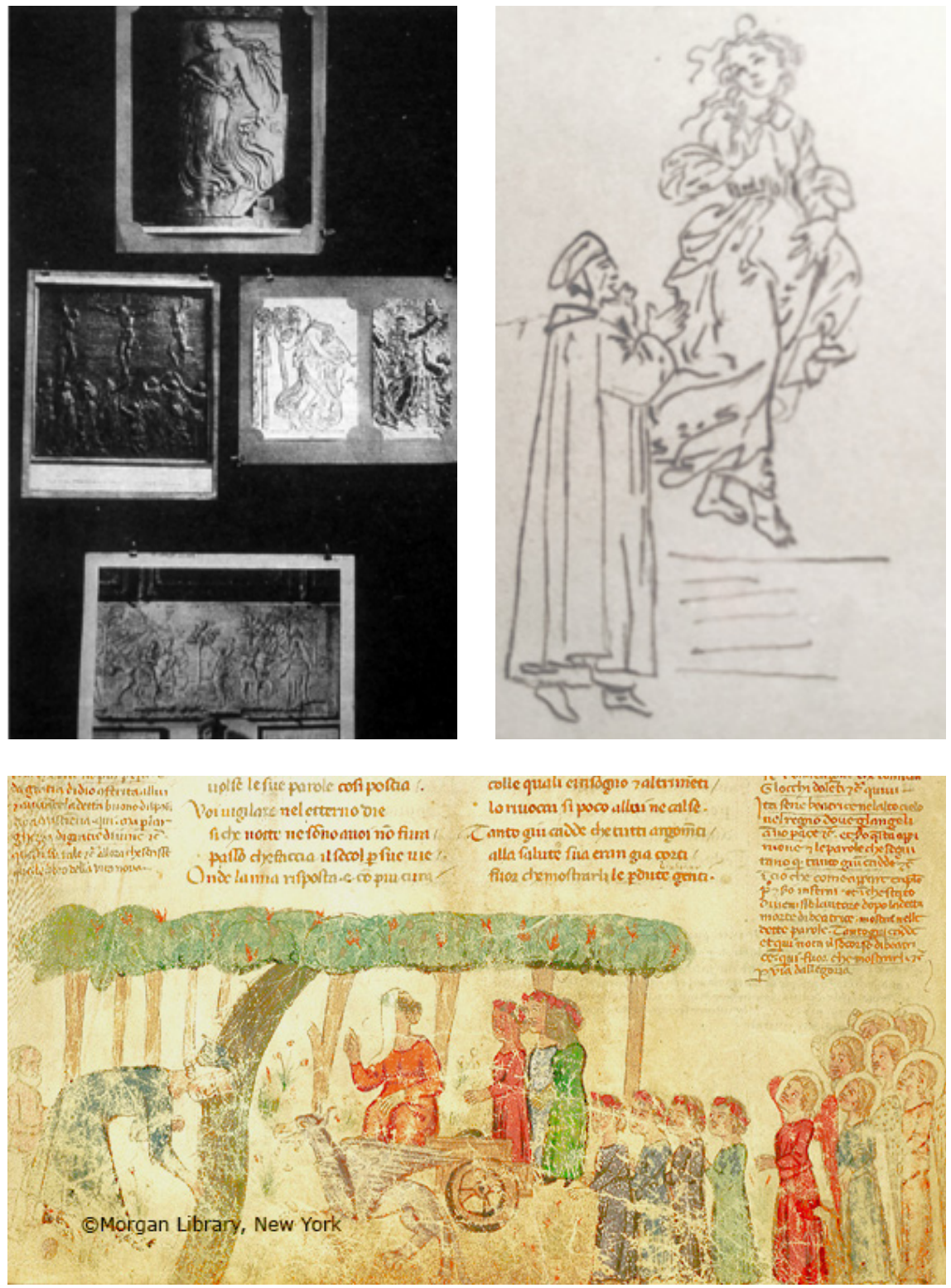

Fig. I3. Aby Warburg, Tavola ninfe, Warburg Institute Archive III. I09.3.

Fig. I4. Aby Warburg, Matelda e Dante, da Botticelli, Commedia, Pg. XXVIII, disegno, Warburg Institute Archive, III.39.5.2, fol.3r.

Fig. I5. Pg. XXX, I2I-I32, Ms. 676, f. 85v. New York, Morgan Library. 

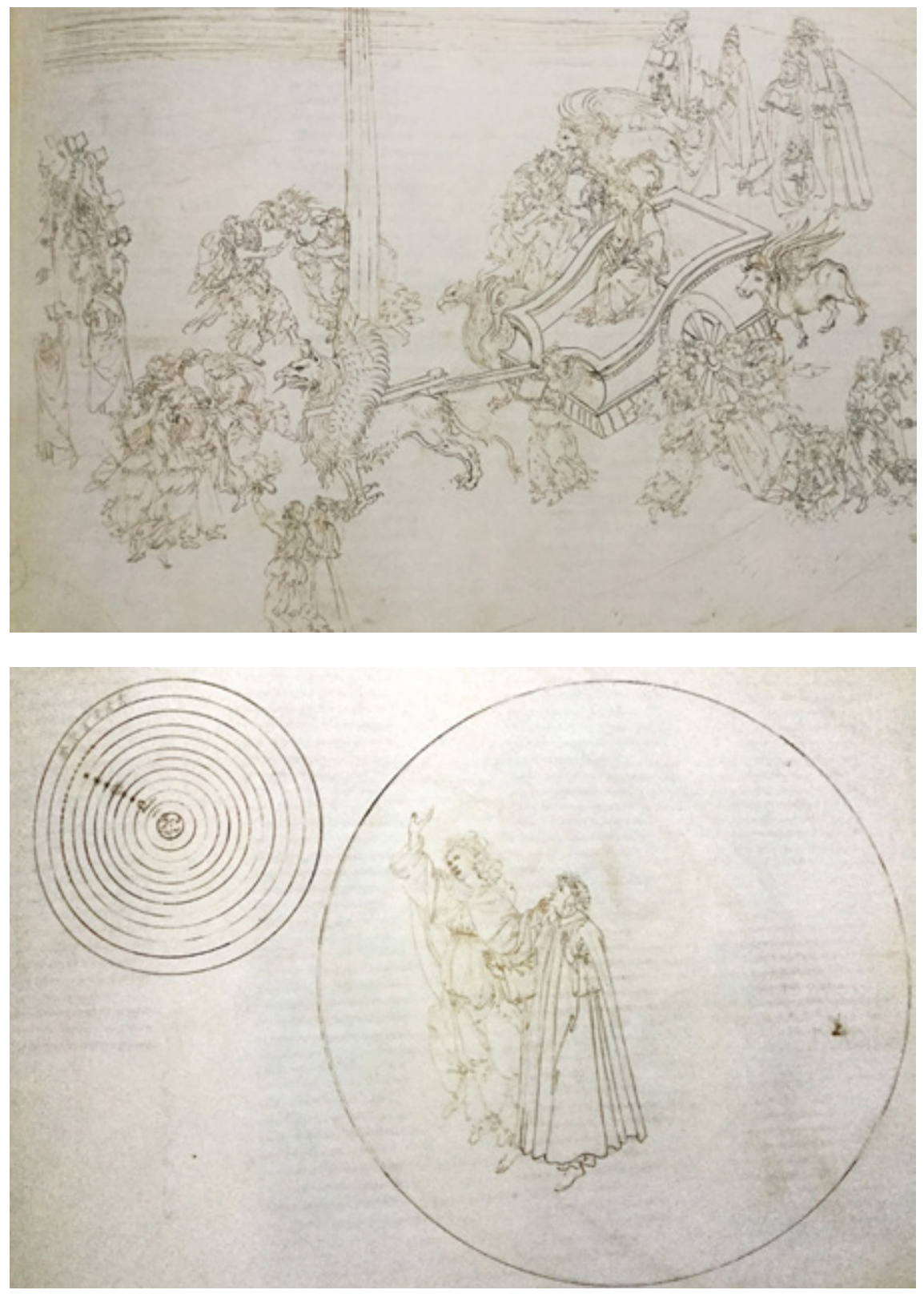

Fig. I6. Pg. XXXI, I06-II4, Botticelli, Ms. Hamilton 20I, Berlino Kupferstichkabinett. Fig. I7. Pd. II, Botticelli, Ms. Hamilton 20I, Berlino Kupferstichkabinett. 

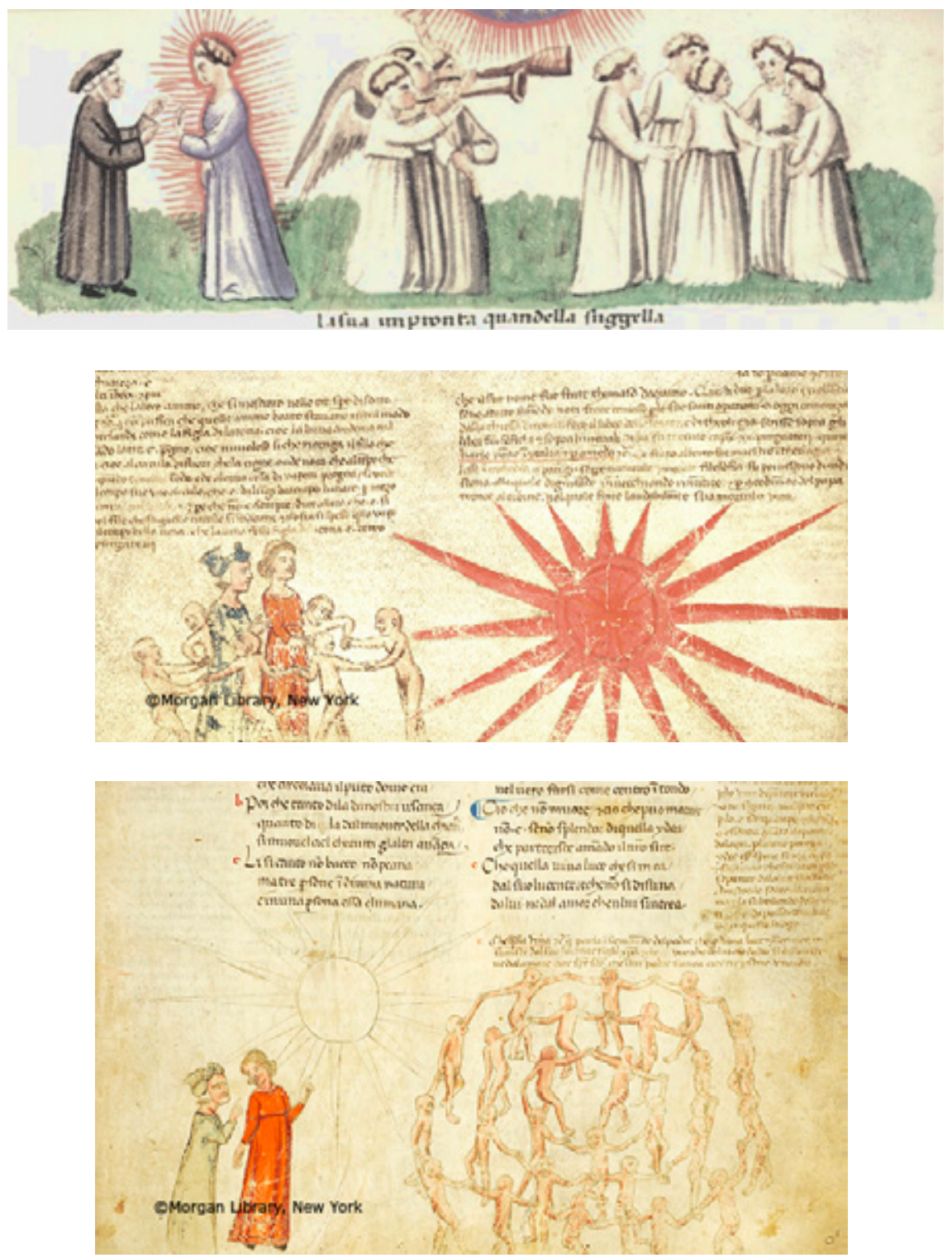

Fig. 18. Pd. VII, 4-9, Ms. it. 474, fol. I03, v., Modena, Biblioteca Estense.

Fig. 19. Pd. X 7, Ms. 676, I02, v. , New York, Morgan Library.

Fig. 20. Pd. XIII, I0-24, M.676, New York, Pierpont Morgan Library. 

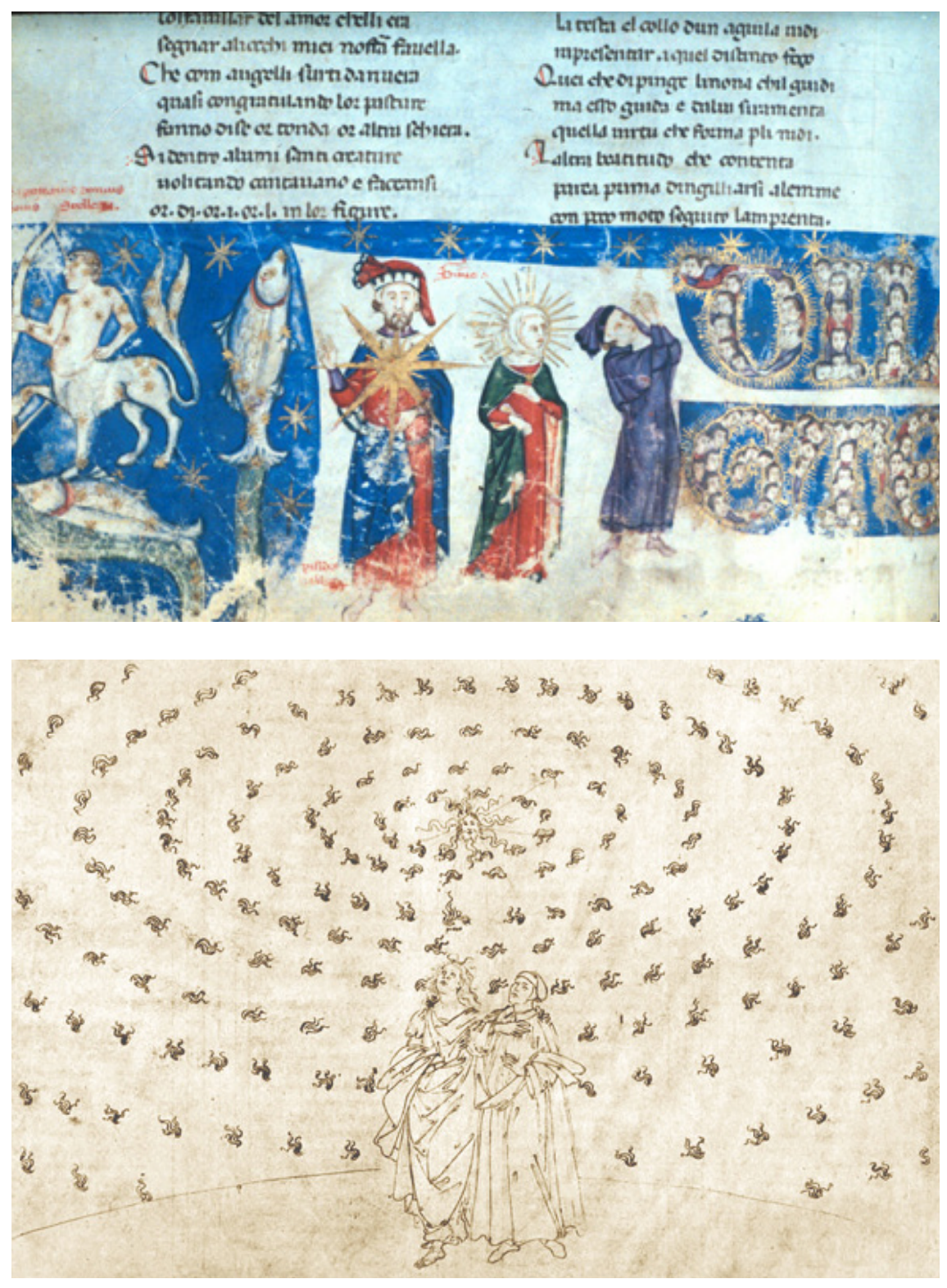

Fig. 2I. Pd. XVIII I, Ms.Holkham 49, fols. I3I-I32, Orford, Bodleian Library.

Fig.22. Pd. XXIV I4, Botticelli, Ms. Hamilton 20I, Berlino Kupferstichkabinett. 


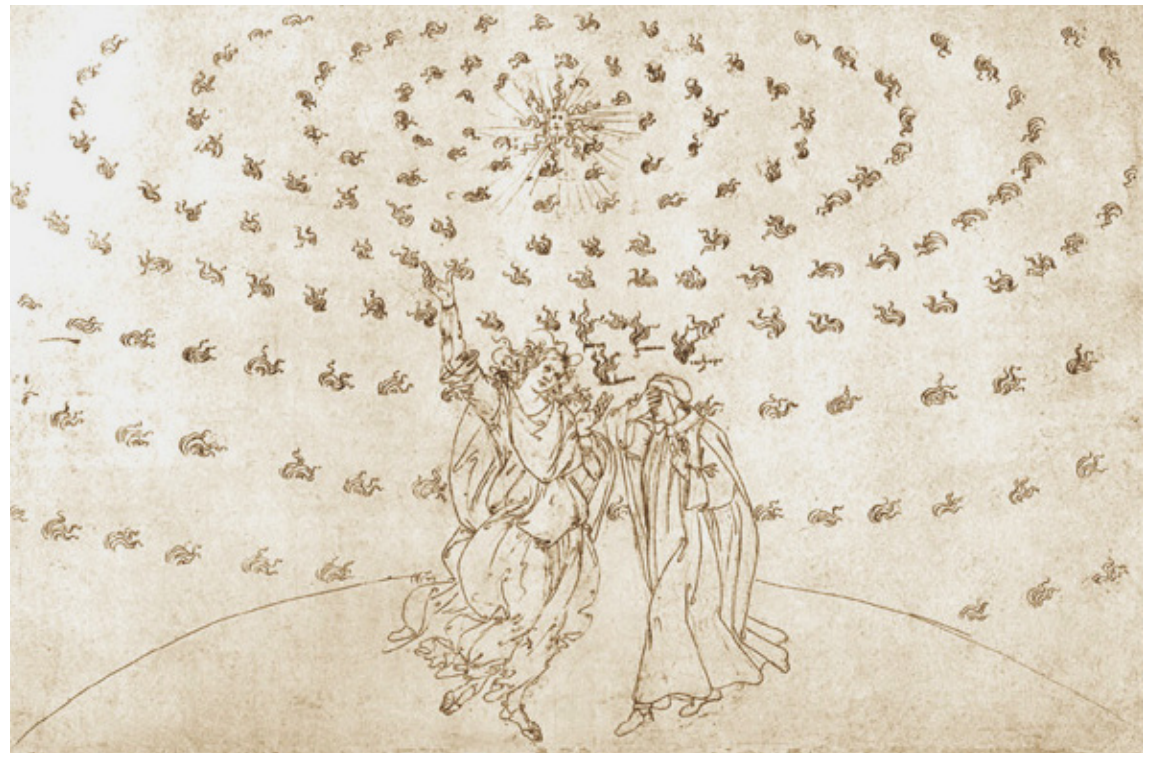

Fig. 23. Pd. XXVI 8 , Botticelli, Ms. Hamilton 20I, Berlino Kupferstichkabinett. 


\section{BIBLIOGRAFIA}

Agamben, G., 2007, Ninfe, Bollati Boringhieri, Torino.

Antonelli, R., 2016, "La Primavera, Warburg e Dante: altre suggestioni?", in Iconologie. Studi in onore di Claudia Cieri Via, a cura di I. Miarelli Mariani, Stefano Pierguidi Marco Ruffini, Roma, Campisano Editore, pp. I3-I9.

Barolsky, P., 1981, "Botticelli’s Primavera", Konsthistorisktskrift Tidskrift, LII, pp. I-6;

Battistini, A., 2008, "Le competenze figurative di Dante", Prefazione a L. Pasquini, Iconografie dantesche: dalla luce del mosaico alle immagini profetiche, Ravenna, Longo Editore, pp. p.8-9.

Bredekamp, H., 1996, Botticelli, La Primavera, (1988), Modena, Franco Cosimo Panini.

Brieger, P., Meiss, M., Singleton, C. S., 1969, Illuminated Manuscript of the Divine Comedy, 2 vols, New York - Princeton, Princeton University Press.

Busch, W., 2009, Aby Warburg und Frederich Nietzsche. Pathosformel und Sprachgebärde in den "Dionysos-Dithyramben", in Ekstatische Kunst - besonnenes Wort, hrsg. Peter Kofler, Bozen, Edition Sturzflüge, pp. 203-216.

Carandini, S., Vaccarino, E. (edd.), I997, La generazione danzante. L'Arte del movimento in Europa nel primo Novecento, a cura di S. Carandini e E. Vaccarino, Roma, Di Giacomo.

Casini Ropa, E., 2007, Danza, Cultura e Società nel Rinascimento italiano, Macerata, Ephemeria Editrice.

Catoni, M.L., 2008, La comunicazione non verbale nella Grecia antica, Torino, Bollati Boringhieri.

Ciccuto, M., 2016, Origini poetiche e figurative di una leggenda dantesca: Matelda nell'Eden, Schuetze-Terzoli 2016, pp. 49-79.

Cieri Via, C. 1990, Note sull'iconografia della danza nel Quattrocento fra movimentoed espressione, in Padovan 1990, pp. 25I-262.

—, 2006, "Aby Warburg e la danza come 'puro atto della metamorfosi”", Quaderni Warburg Italia, 2-3, pp. 63-136.

—, 20II, Il sacrificio di Polissena come rituale del sangue. Aby Warburg e la sopravvivenza di un topos antico, in Homère à la Renaissance, Sous la dir. de Luisa Capodieci et Philip Ford, Paris-Roma, Somogy-Académie de France, pp. I05-I24.

—, 20II, "La Commedia di Dante in immagine nell'arte del Rinascimento", Critica del testo. Dante Oggi/ 2, a cura di R. Antonelli, A. Landolfi, A. Punzi, pp. 58I-6I2.

—, "Visibile parlare. Ombre e immagini silenziose nella Commedia dantesca", Dante and the Visual Arts Symposium, The Getty Center and UCLA Humanities, 2I-23 August 2016, Artibus et Historiae, c. d. s.

Ciocciola, C., 1989, "Visibile parlare", Rivista di Letteratura italiana, VIII, pp. 9-77.

Clark, T. (ed.), 20I7, Hokusai beyond the great Wave, ed. by Timothy Clark, London, British Museum.

Di Rienzo, C., 20I6, "La danza e la grazia", Agalma, 3I, pp. 34-47.

Didi-Huberman, G., 20I5, Ninfa fluida. Essai sur le drapé desir, Paris, Gallimard.

Francalanci, A., 1992, "Le tre Grazie della Primavera del Botticelli: La danza fra allegoria e realtà storica", Medioevo e Rinascimento. Annuario del Dipartimento di studi sul Medioevo e il Rinascimento dell'Università di Firenze, VI n.s. III, pp. 23-37.

Franzoni, C, 2006, Danzare i gesti. "Recensione" a: Maria Luisa Catoni, Schemata. Comunicazione non verbale nella Grecia antica, Pisa, Edizioni della Normale, 2005, Engramma, 46. 
Fratini, R., 20I4, "Vacabimus. Appunti per una cinesiologia dantesca", in Danses imaginades, danses relatades. Paradigmes iconogràfics del ball des de l'antiguitat clàssica fins a l'edat mitjana, a cura di L. Buttà, J. Carruesco, F. Massip, E. Subías, Tarragona, Institut Català d'Arqueologia Clàssica, pp. 85-Io8.

Freud, S., I977, Saggi sull'arte e la letteratura, Torino, Bollati Boringhieri.

Goethe, J. W., I994, Laocoonte e altri scritti sull'arte (I789-I805), Roma, Salerno.

Guglielmo Ebreo, De practica seu arte tripudi, del I463, Paris, Bibliothèque Nationale, f. ital. 973 .

Inglese, G. (ed.), 2016 = Alighieri, Dante Commedia. Purgatorio, a cura di Giorgio Inglese, Roma, Carocci.

—, (ed.), 2or6a, Alighieri, Dante, Commedia. Paradiso, a cura di di Giorgio Inglese, Roma, Carocci Editore.

La Malfa, C., I999, "Firenze e l'allegoria dell'eloquenza: Una nuova interpretazione della Primavera di Botticelli", Storia dell'arte, 97, pp. 249-293.

Camesasca, E., 200o, Leonardo, Trattato della Pittura, ed. a cura di E. Camesasca, Vicenza, Neri Pozza.

Mallé, L. (ed.), I95I, Alberti, L.B. Della pittura, Edizione critica a cura di L. Mallé, Firenze, Sansoni.

Mancini, L., 2006, "La danza per 'figure'. Immagini del movimento ritmico nella Grecia arcaica”, Quaderni Warburg Italia, 2-3, pp. I53-194.

Manning, S., 2016, Mary Wigmann e la danza tedesca nel primo Novecento, Roma, Istituto tedesco.

Meyer-Baer, K., I970, The Music in Dante's Cosmos, Appendice III in Music of the Spheres and the Dance of Death, Princeton University Press, pp. 352-356.

Migliorini Fissi, R., 2008, "L'incontro di Dante con Matelda, la "bella donna", del Paradiso terrestre", Letture classensi, 35-36, "Dante e l'Arte" a cura di Claudia Giuliani, Longo Editore, Ravenna, pp. I05-I28.

Millepied, G., Vigarello, G., Martinez, J.L. (edd.), 2016, Corps en mouvement. La danse au Musée, par B. Millepied, G. Vigarello, J-L. Martinez, Paris, Musée du Louvre.

Nassar, E. P., I994, Illustrations to Dante's Inferno, Rutherford, Fairleigh Dickinson University Press.

Padovan, M. (ed.), I990, Guglielmo Ebreo da Pesaro e la danza nelle corti italiane del $X V$ secolo, a cura di M. Padovan, Pisa, Pacini, I990.

-, 1987, La danza alle corti italiane del XV secolo: arte figurativa e fonti storiche, in Mesura e Arte del danzare: Guglielmo Ebreo da Pesaro e la danza nelle corti italiane del XV secolo, Pesaro, pp. 50-II2.

Panofsky, E., Saxl, F., 2009, Mitologie classiche nell'arte medievale, Torino, Aragno.

Pasquino P., 20I7. Benvenuto da Imola: "Lectura Dantis Bononiensis", Ravenna, Longo. Petersen, E., I917, Rhythmus, Berlin, Weidmann.

Pietrini, S., 2000, "La santa danza di David e il ballo peccaminoso di Salomé. Due figure esemplari nell'immaginario biblico medievale", Quaderni medievali, 50, pp. 45-59.

Pontemoli, A., 2009, "La danza di Domenico da Piacenza", in Il mondo cortese di Gentile da Fabriano e l'immaginario musicale. La cultura musicale e artistica nel Quattrocento europeo e la sua riscoperta in epoca moderna e contemporanea, a cura di M. Lacché, Roma, Aracne Editore, pp. 57-77.

Pucci, G., 2005, "Il rhythmos nell'arte figurativa greca”, Quaderni Warburg Italia, 2-3, pp. I37-I52. 
Santarelli, C., 2004, "Tipologie di re David nei codici miniati medievali", in Prospettive di iconografia musicale, a cura di N. Guidobaldi, Milano, Associazione culturale Mimesis, pp. I09-I28.

Sapegno, N. (Ed.), 1985 = Alighieri, Dante, La Divina Commedia, Il Purgatorio, a cura di Natalino Sapegno, Firenze, LaNuova Italia.

Saxl, F., 20I2, "I gesti espressivi nell'arte figurativa" (I932), a cura di Claudia Cieri Via, Annali di Critica d'arte, VIII, pp. I-25.

Schuetze, S., Terzoli, M. A. (edd.), 20I6, Dante und die bildenden Kuenste, Berlin Boston, De Gruyter.

Seznec, J., I98I, La sopravvivenza degli antichi dei, Torino, Bollati Boringheri.

Sparti, B., 2003, "Humanism and the Arts. parallels between Alberti's On Painting and Guglielmo Ebreo's On Dancing", in Art and music in the early modern period, ed. By K. McIver, Aldershot, pp. I73-I92.

Stoichita, V., 20Io, L'effetto Pigmalione, Milano, Il Saggiatore.

Terzi A., 2003, Il commento di Iacopo della Lana alla Commedia: l'Inferno secondo il ms. Riccardiano I005: lettura e testo, Roma, Università degli Studi di Roma "La Sapienza”, Tesi di Dottorato, XVI ciclo 2002-2003.

Terzoli, A., 20I6a, "Visibile parlare: ecfrasi e scrittura nella Commedia, Dante und die bildenden Kuenste", in Schuetze -Terzoli 2016, pp. 23-48.

Venturi, G., 2006, Una "Lectura Dantis" e l'uso dell'ecfrasi: Purgatorio X, in Ecfrasi. Modelli d'esempi fra Medioevo e Rinascimento, a cura di Gianni Venturi e Monica Farneti, Bulzoni Editore, pp. I5-32.

Warburg, A., 20II, Frammenti sull'espressione, a cura di Susanne Mueller, Pisa, Edizioni della Normale.

Wilson, T. (ed.), I988, Domenico da Piacenza, De la arte di ballare e danzare, Ms. ital. 972, a cura di D. Wilson, Cambridge, Early dance circle.

Winckelman, J.J., 2008, Della grazia nelle opere d'arte (1759), in J.J. Winckelman, Il bello nell'arte. La natura gli antichi, la modernità, Nuova edizione a cura di C. Franzoni, Torino, Einaudi, pp. 53-59. 
